\title{
Reagent Reactivity and Solvent Choice Determine Metal- Organic Framework Microstructure During Postsynthetic Modification
}

\author{
Derek R. Du Bois ${ }^{\mathrm{a}}$ and Adam J. Matzger*a,b \\ ${ }^{\mathrm{a}}$ Department of Chemistry and ${ }^{\mathrm{b}}$ Macromolecular Science and Engineering Program, University of \\ Michigan, 930 North University Avenue, Ann Arbor, Michigan 48109-1055, United States
}

\section{Supporting Information}

Table of Contents

I. Materials and Methods

II. MOF Synthesis

III. Postsynthetic Modification Reactions

IV. Experimental Scanning Electron Microscopy and Energy-Dispersive X-Ray Spectroscopy Data

V. Experimental Infrared Kinetics Data

VI. Experimental ${ }^{1} \mathrm{H}$ Nuclear Magnetic Resonance Spectroscopy Data

VII. Plots of PSM Conversion Calculated from ${ }^{1} \mathrm{H}$ NMR Data Over Time

VIII. Additional SEM-EDS Data

IX. References 


\section{Materials and Methods}

Chemicals were used as purchased without purification unless otherwise noted. 2-Aminoterepthalic acid, 4-bromophenyl isocyanate, chloroacetyl isocyanate, 2-chloroethyl isocyanate, deuterium chloride $35 \%$ in $\mathrm{D}_{2} \mathrm{O}$, and 1,2-dichloroethane were purchased from Sigma Aldrich. Anhydrous $N, N$-dimethyl formamide (DMF) purchased from Sigma Aldrich was stored over activated $3 \AA$ molecular sieves in a glove box where it was kept for 3 to 4 days before use. Dry acetonitrile, dry chloroform stabilized with amylene, DMF dried over $4 \AA$ molecular sieves, anhydrous toluene, and zinc nitrate hexahydrate were purchased from Fisher Scientific. Trifluoroacetyl isocyanate was purchased from Enamine. Butyl anthranilate was purchased from TCI chemicals. Carbon tetrachloride and methyl sulfoxide- $\mathrm{d}_{6}$ were purchased from Acros Organics. Trichloroethylene was purchased from Alfa Aesar. Chloroform-D + silver foil was purchased from Cambridge Isotope Laboratories, Inc. JEOL aluminum specimen mounts were used with PELCO carbon conductive tabs, both from Ted Pella Inc., as stages for scanning electron microscopy (SEM) in conjunction with energy-dispersive X-ray spectroscopy (EDS). A JEOL JSM-7800FLV scanning electron microscope operating with an accelerating voltage of $20 \mathrm{kV}$ was used for SEM-EDS experiments. After PSM, IRMOF3 samples were prepared for SEM-EDS analysis by sectioning crystals in toluene parallel to the 100 plane using a razor blade and placing the crystal cross sections with the cut side up on the carbon tape adhered to the aluminum stage, followed by activation under vacuum. Mid-infrared spectroscopy was performed using a Thermo Scientific Nicolet iS50 FT-IR in transmission mode with a SmartSeal Trans Cell with $0.05 \mathrm{~mm}$ $\mathrm{CaF}_{2}$ windows made by PIKE Technologies. ${ }^{1} \mathrm{H}$ NMR spectra were recorded on a Varian vnmrs $500 \mathrm{MHz}$ spectrometer. For each ${ }^{1} \mathrm{H}$ NMR experiment, approximately $8.6 \mathrm{mg}$ of IRMOF-3 modified with either 2chloroethyl isocyanate or chloroacetyl isocyanate were digested by sonication in $600 \mu \mathrm{L}$ of DMSO- $\mathrm{d}_{6}$ and $2.3 \mu \mathrm{L}$ of $35 \%$ deuterium chloride in $\mathrm{D}_{2} \mathrm{O}$. Upon dissolution of the crystals, this solution was filtered through a $0.45 \mu \mathrm{m}$ Nylon filter and used for ${ }^{1} \mathrm{H}$ NMR analysis.

\section{MOF Synthesis}

IRMOF-3 was synthesized using a modified literature procedure. ${ }^{1} \mathrm{Zn}\left(\mathrm{NO}_{2}\right)_{3} \cdot 6 \mathrm{H}_{2} \mathrm{O}(478 \mathrm{mg}, 1.61 \mathrm{mmol})$ and 2-aminobenzenedicarboxylic acid $(100 \mathrm{mg}, 0.552 \mathrm{mmol})$ were dissolved in $20 \mathrm{~mL}$ of DMF. The solution was divided into $2 \mathrm{~mL}$ portions and transferred into 10 vials ( 1 dram, $3.7 \mathrm{~mL}$ capacity). The vials were placed in an oven set to $100{ }^{\circ} \mathrm{C}$. The temperature was held overnight, after which the oven was turned off and left to cool with the door slightly ajar for 20-30 minutes. The mother liquor from each vial was pipetted out, and the crystals were washed with DMF $(3 \times 3 \mathrm{~mL})$. The crystals were then washed in toluene or chloroform $(3 \times 3 \mathrm{~mL})$ to reflect the post-synthetic modification (PSM) solution conditions used next. The average yield of dried IRMOF-3 per vial was determined to be approximately $8.6 \mathrm{mg}$ ( $\sim 57 \%$ yield). 


\section{PSM Reactions}

Each PSM reaction was performed on $\sim 8.6 \mathrm{mg}$ of IRMOF-3 using $3 \mathrm{~mL}$ of reaction solution in a 1 dram vial. For analysis by SEM-EDS, each vial of functionalized IRMOF-3 was washed in toluene $(3 \times 3 \mathrm{~mL})$ as the final step before cutting and activating the crystals. Toluene was chosen as the solvent to cut crystals from, because it has sufficiently low volatility to allow the sectioning of crystals with a razor before complete evaporation (allowing for cleaner cuts) and also does not leave trace chlorine to interfere with SEM-EDS.

PSM with either chloroacetyl isocyanate or 2-chloroethyl isocyanate was conducted at $50 \mathrm{mM}$ in either chloroform or toluene. These reactions progressed for 3, 6, 9, or 24 hours before the reaction solution was pipetted out and the crystals were washed in the solvent used for PSM $(3 \times 3 \mathrm{~mL})$. For conditions involving washes with chloroform, toluene was also used to wash the crystals $(3 \times 3 \mathrm{~mL})$. For PSM conditions using only 4-bromophenyl isocyanate as the reagent, $50 \mathrm{mM}$ of 4-bromophenyl isocyanate were used in either chloroform, toluene, a 1:1 mixture of chloroform and toluene, or a 1:5 mixture of anhydrous DMF (to prevent reagent consumption due to residual water) and toluene. Dry DMF was used after PSM to wash the crystals $(3 \times 3 \mathrm{~mL})$. The DMF washing step was followed by washes in toluene $(3 \times 3 \mathrm{~mL})$. For PSM of IRMOF-3 to form a dual functionalized core-shell structure, a solution containing $50 \mathrm{mM}$ each of 4bromophenyl isocyanate and chloroacetyl isocyanate in chloroform was used. After 1 hour, the solution was pipetted out, and the functionalized crystals were washed in chloroform $(3 \times 3 \mathrm{~mL})$ and then toluene $(3 \times 3 \mathrm{~mL})$. For PSM of IRMOF-3 to form a Matryoshka MOF with 3 separate functionalities, a two-step reaction process was used wherein $50 \mathrm{mM}$ of trifluoroacetyl isocyanate in fresh deuterated chloroform was used in the first step for 16 hours, followed by washing in chloroform $(3 \times 3 \mathrm{~mL})$. In the second step, 50 $\mathrm{mM}$ of 4-bromophenyl isocyanate and $5 \mathrm{mM}$ of chloroacetyl isocyanate in choloroform were used for PSM over 21 hours, followed by washing in chloroform $(3 \times 3 \mathrm{~mL})$ and then toluene $(3 \times 3 \mathrm{~mL})$. 


\section{Experimental SEM-EDS Data}
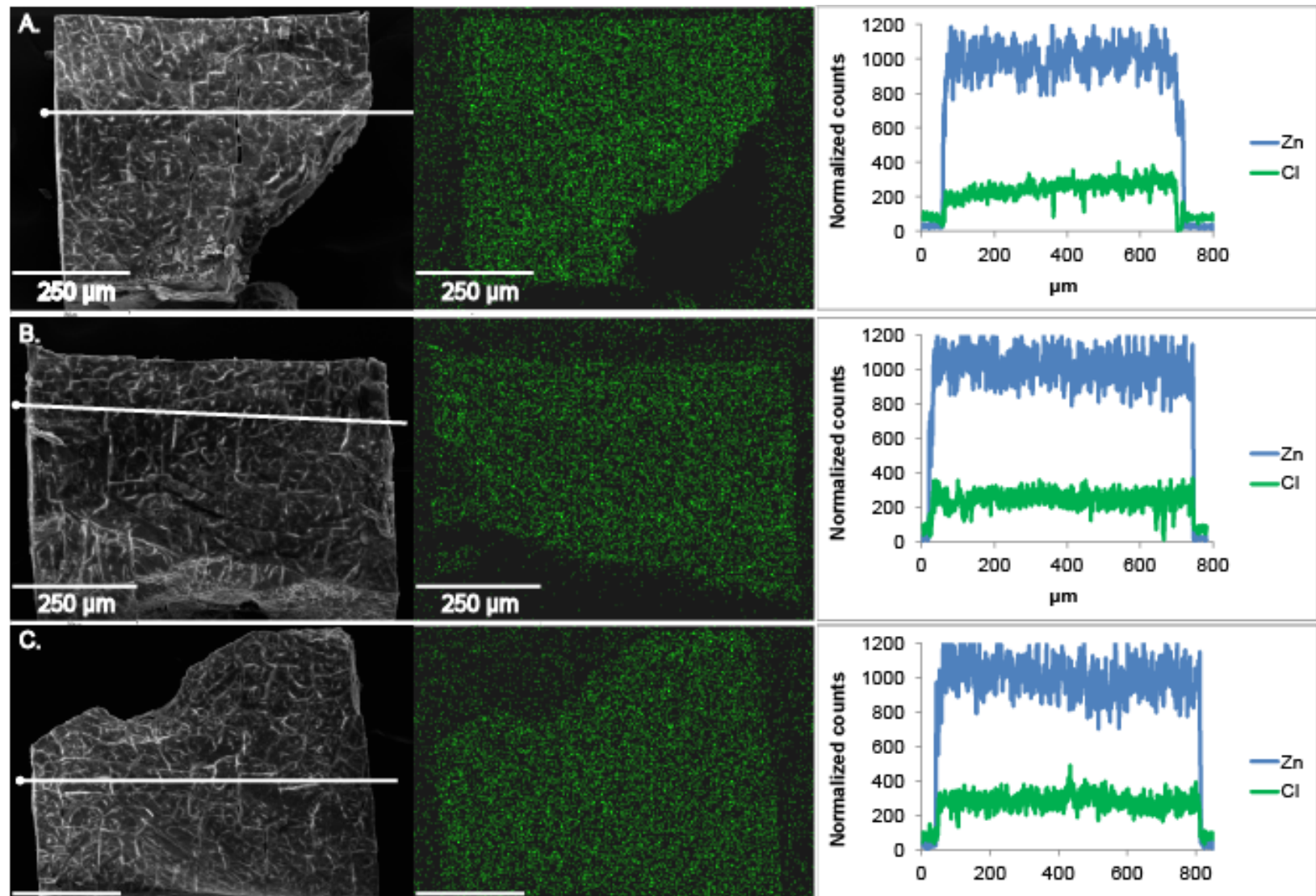

$250 \mu \mathrm{m}$
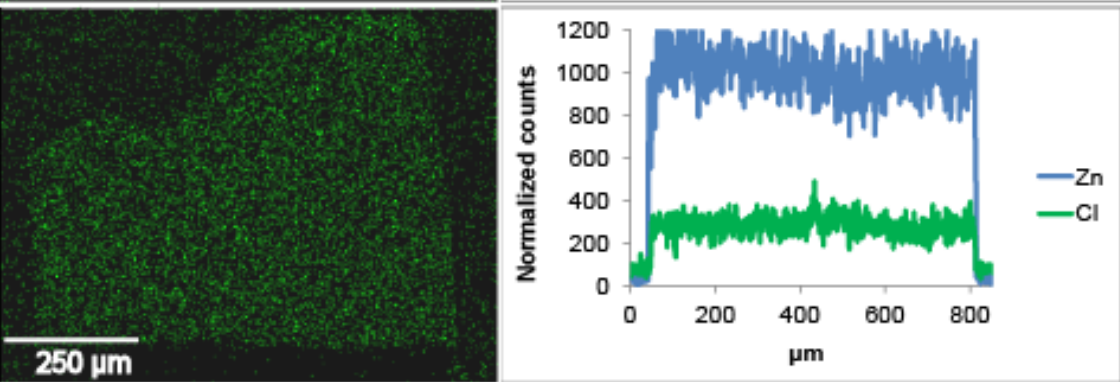

Figure S1. SEM images, EDS maps, and EDS linescans for three trials of PSM on IRMOF-3 with 2chloroethyl isocyanate for 3 hours in toluene, resulting in a uniform microstructure. Zinc counts on linescans normalized to average 1000 . 


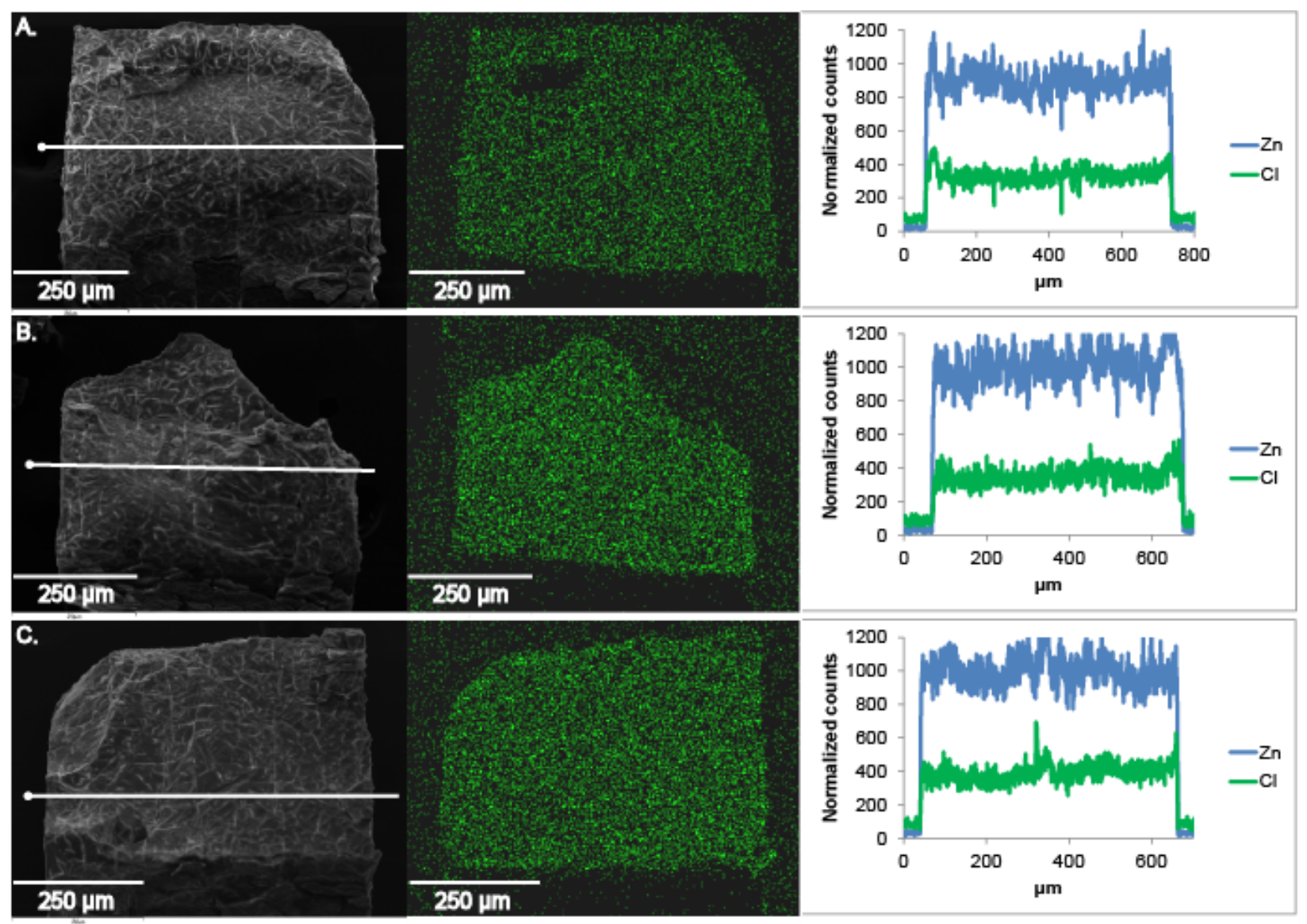

Figure S2. SEM images, EDS maps, and EDS linescans for three trials of PSM on IRMOF-3 with 2chloroethyl isocyanate for 6 hours in toluene, resulting in a uniform microstructure. Zinc counts on linescans normalized to average 1000 . 


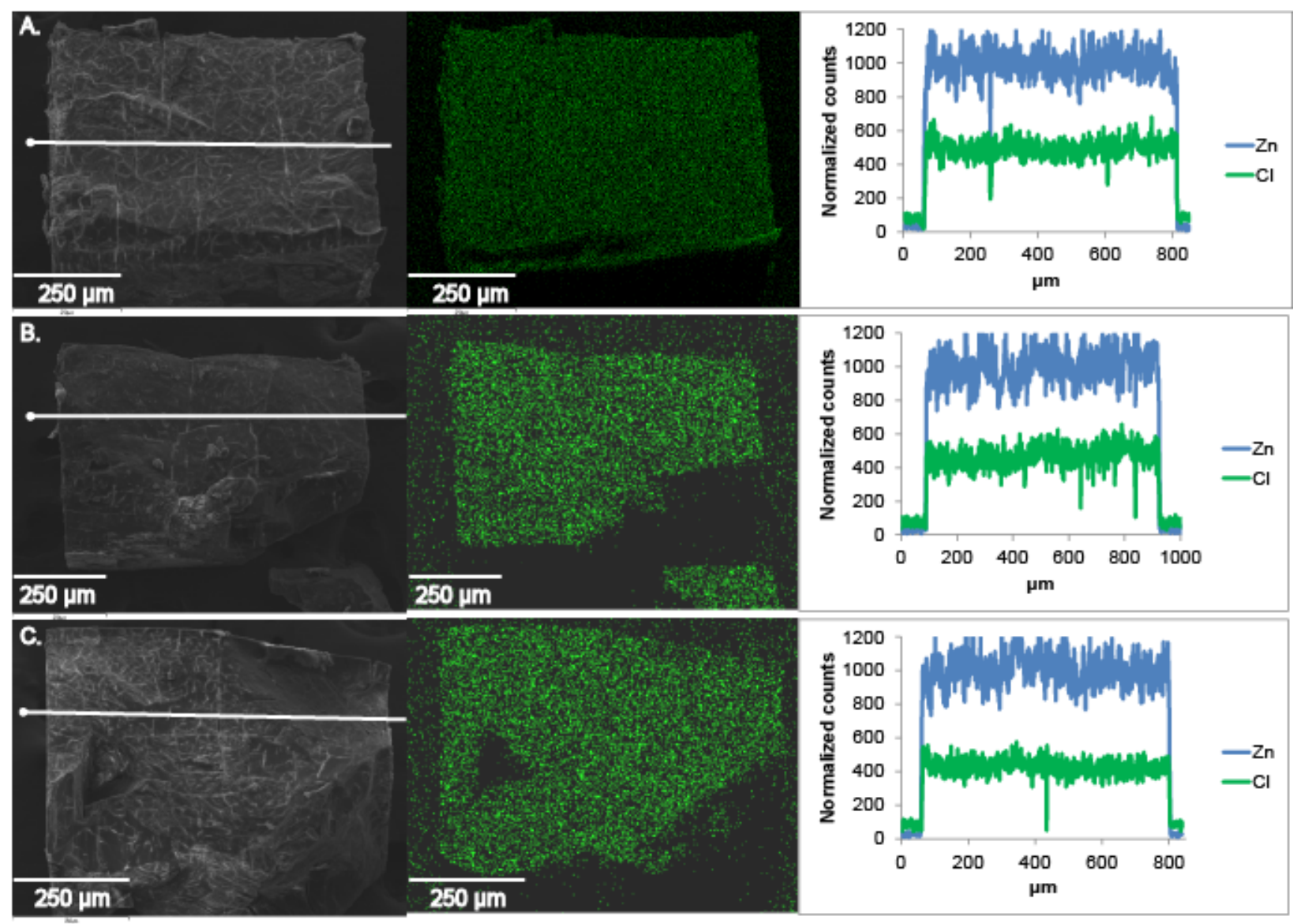

Figure S3. SEM images, EDS maps, and EDS linescans for three trials of PSM on IRMOF-3 with 2chloroethyl isocyanate for 9 hours in toluene, resulting in a uniform microstructure. Zinc counts on linescans normalized to average 1000. 


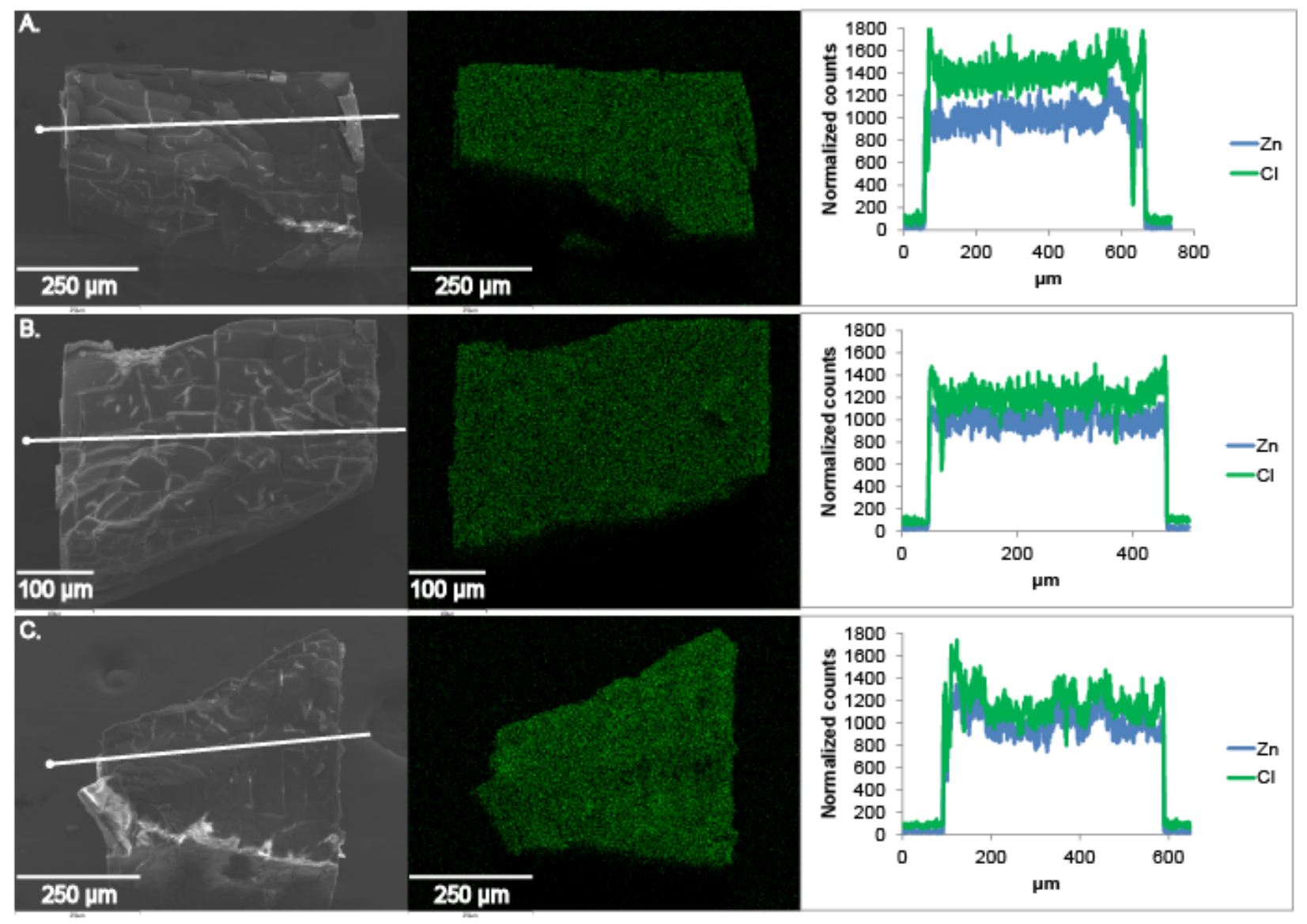

Figure S4. SEM images, EDS maps, and EDS linescans for three trials of PSM on IRMOF-3 with 2chloroethyl isocyanate for 24 hours in toluene, resulting in a uniform microstructure. Zinc counts on linescans normalized to average 1000. 


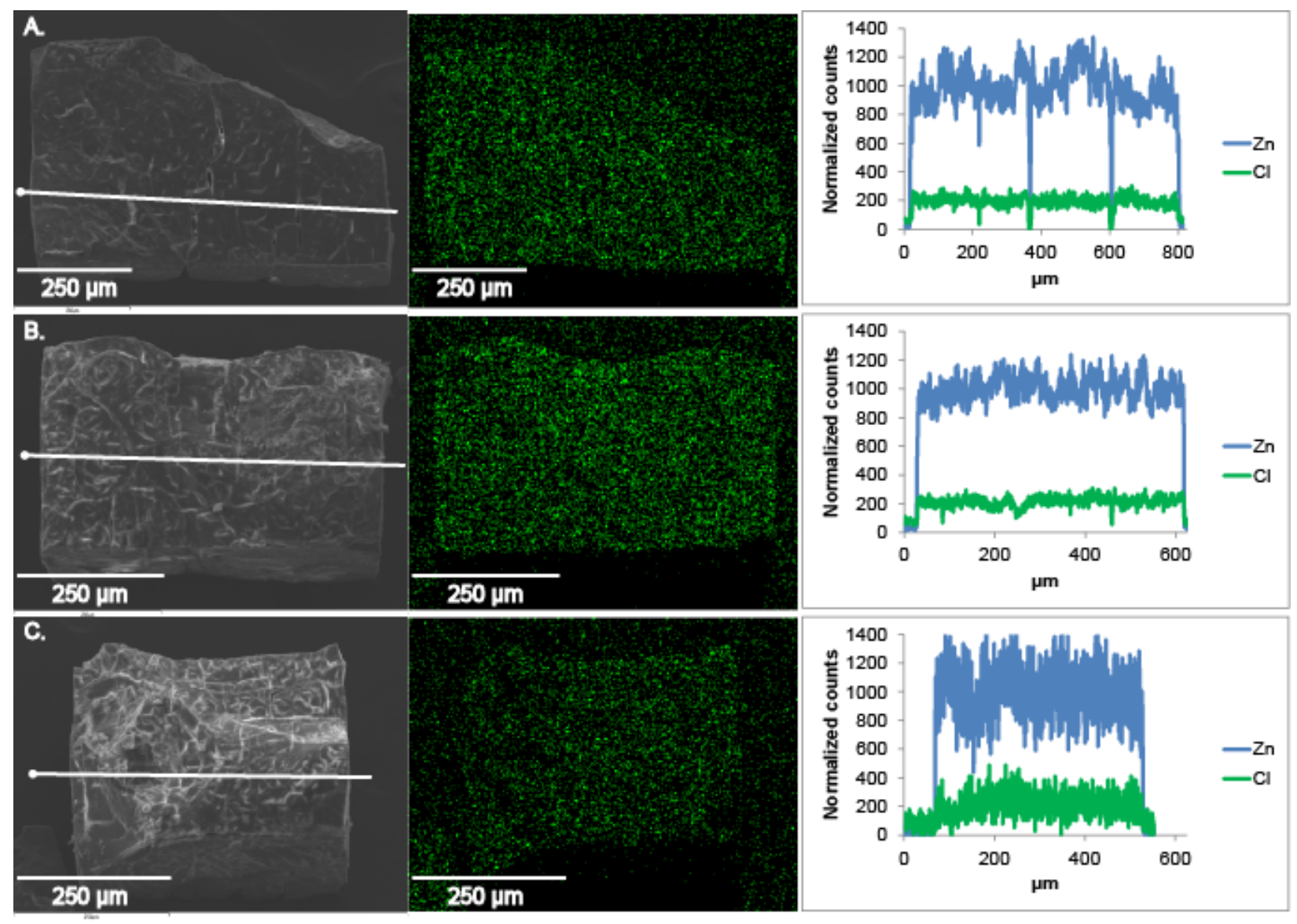

Figure S5. SEM images, EDS maps, and EDS linescans for three trials of PSM on IRMOF-3 with 2chloroethyl isocyanate for 3 hours in chloroform, resulting in a uniform microstructure. Zinc counts on linescans normalized to average 1000. 

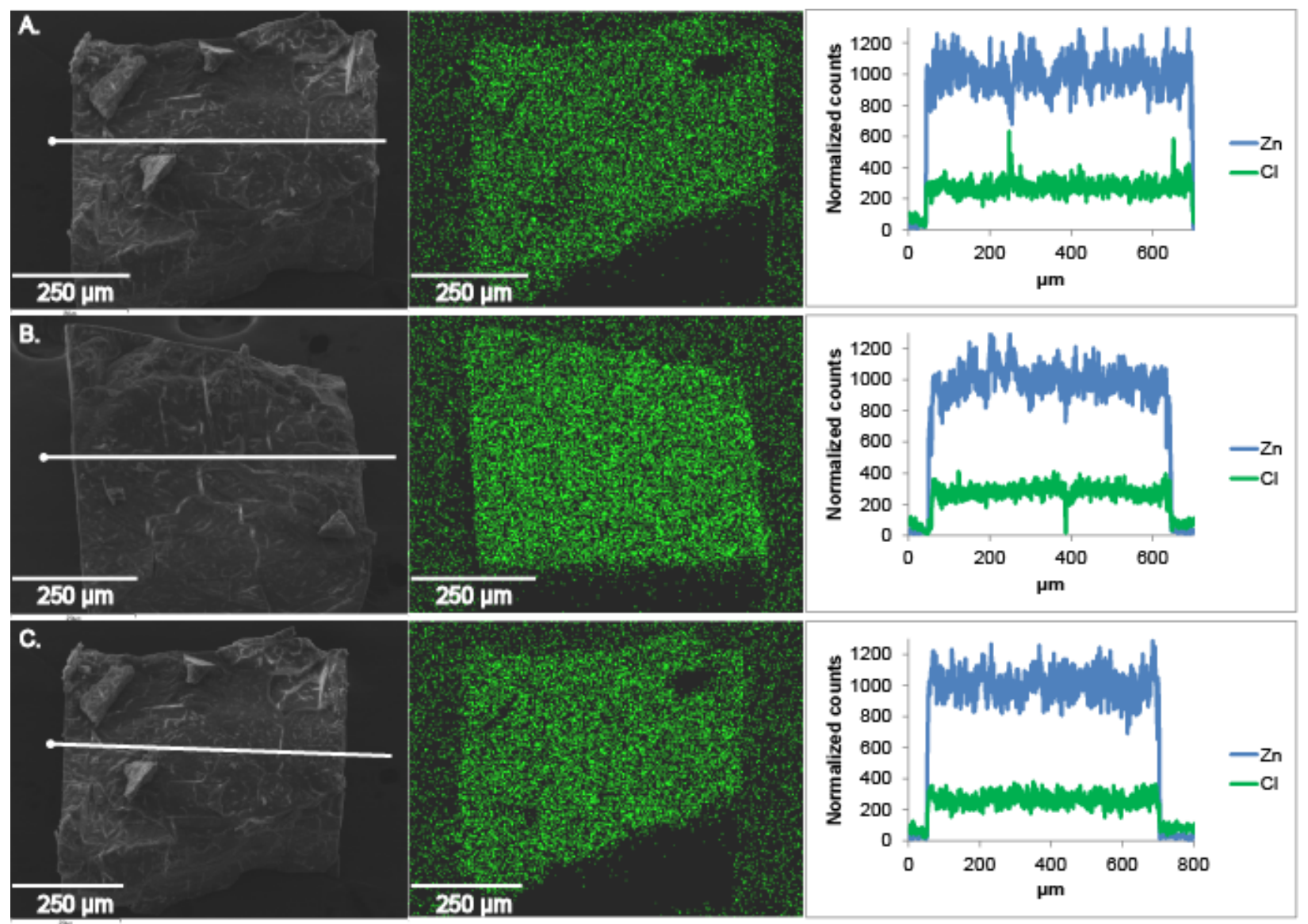

Figure S6. SEM images, EDS maps, and EDS linescans for three trials of PSM on IRMOF-3 with 2chloroethyl isocyanate for 6 hours in chloroform, resulting in a uniform microstructure. Zinc counts on linescans normalized to average 1000. 


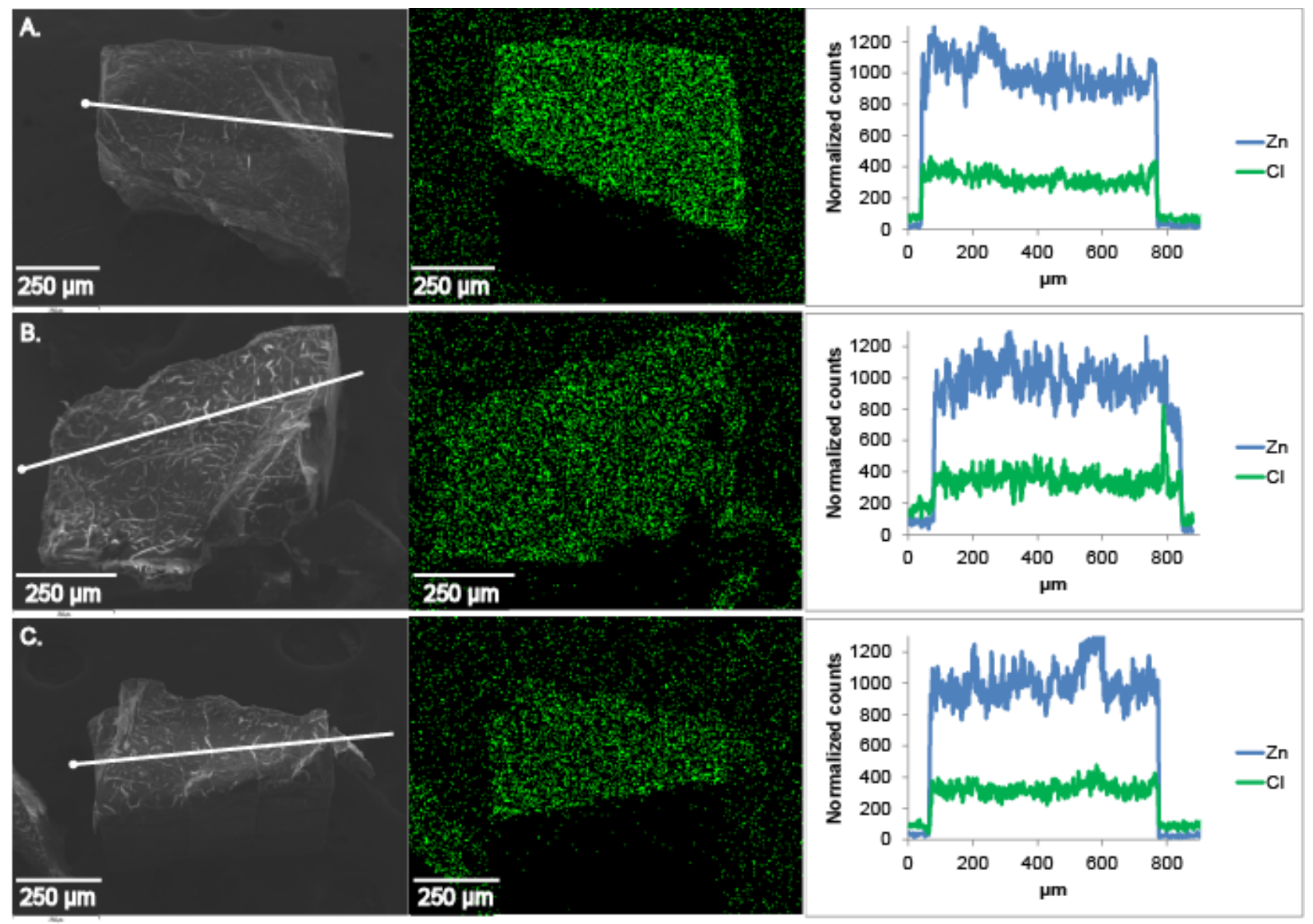

Figure S7. SEM images, EDS maps, and EDS linescans for three trials of PSM on IRMOF-3 with 2chloroethyl isocyanate for 9 hours in chloroform, resulting in a uniform microstructure. Zinc counts on linescans normalized to average 1000. 

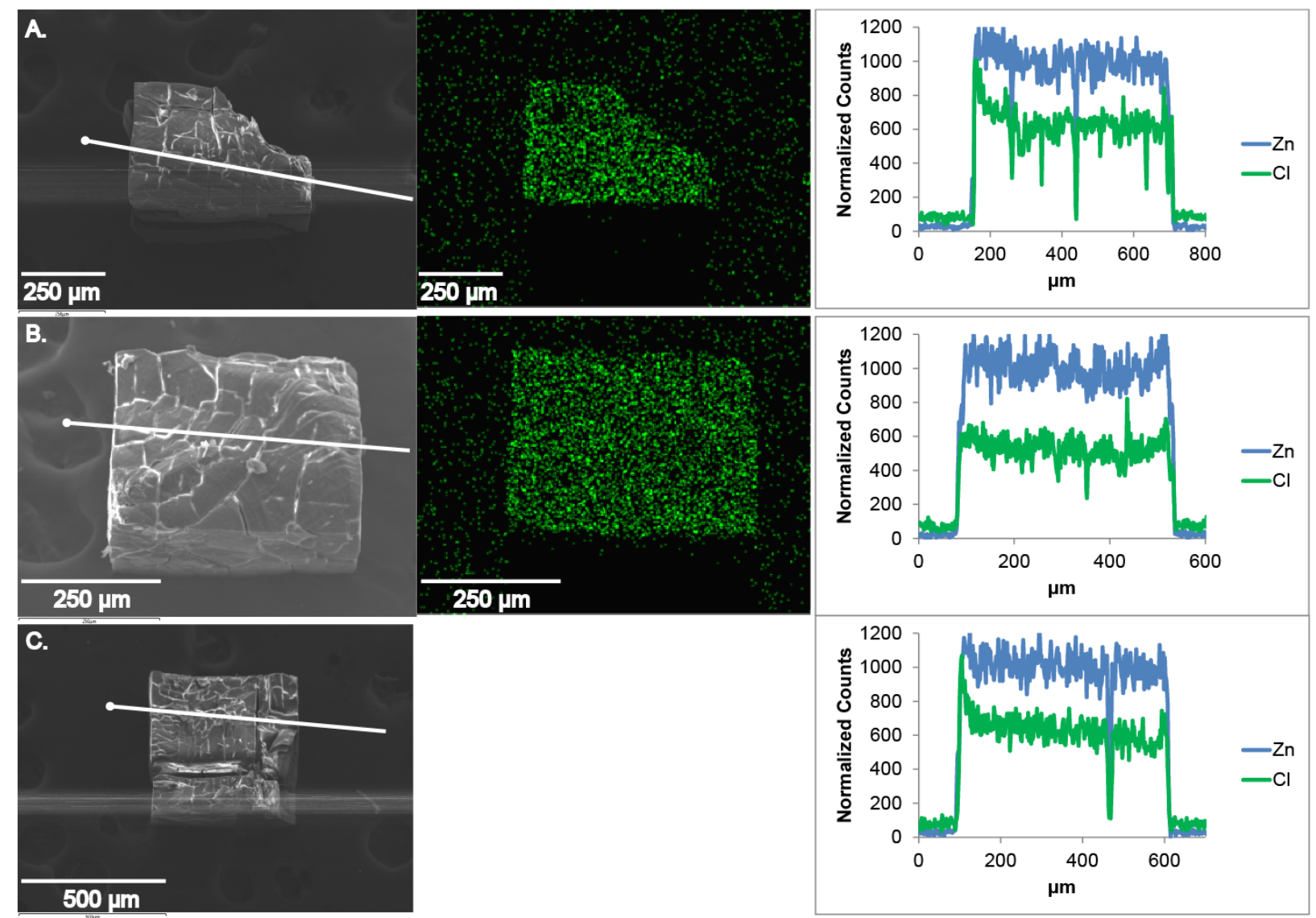

Figure S8. SEM images, EDS maps, and EDS linescans for three trials of PSM on IRMOF-3 with 2chloroethyl isocyanate for 24 hours in chloroform, resulting in a uniform microstructure. Zinc counts on linescans normalized to average 1000. EDS mapping data was not collected for trial $\mathrm{c}$. 


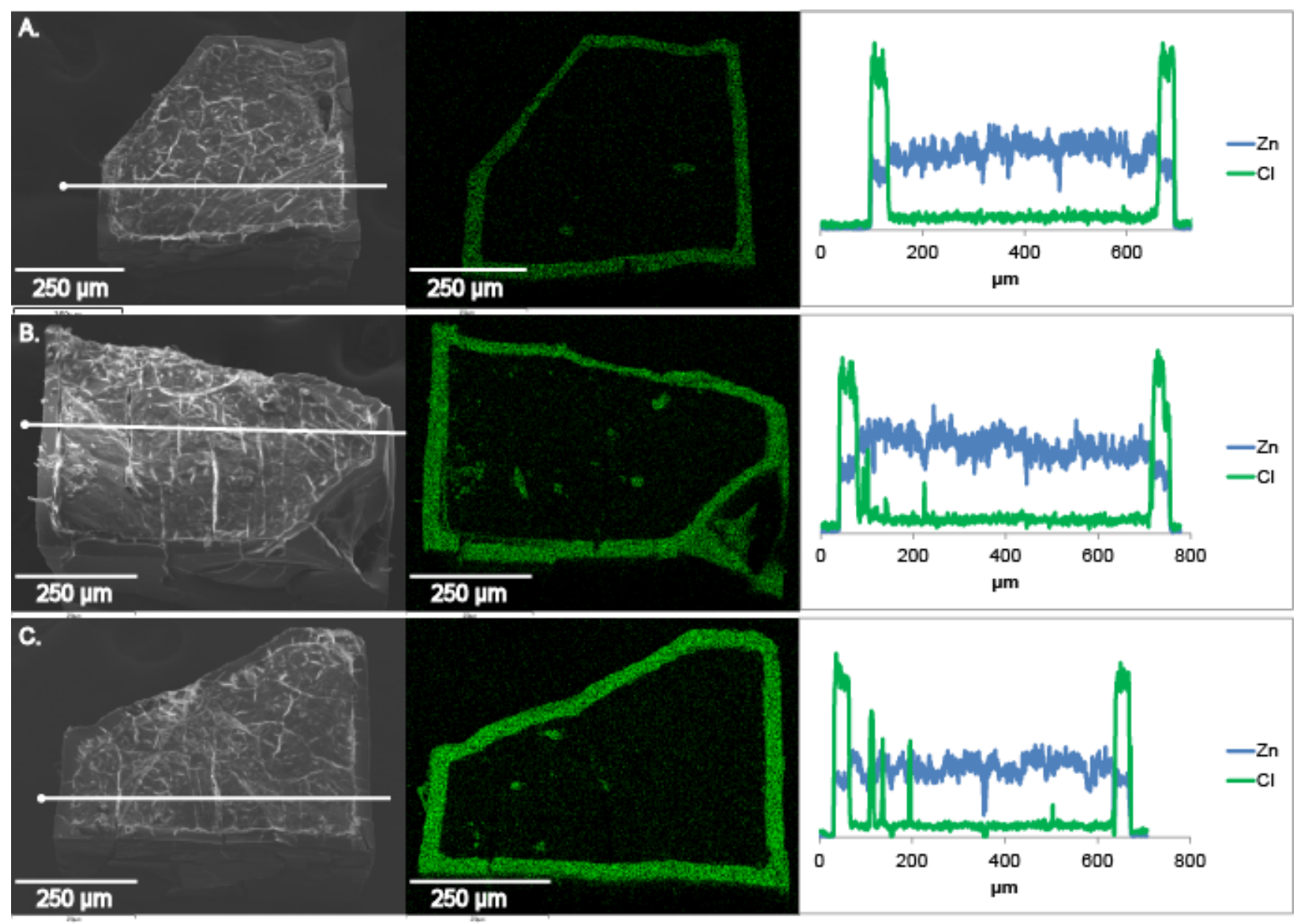

Figure S9. SEM images, EDS maps, and EDS linescans for three trials of PSM on IRMOF-3 with chloroacetyl isocyanate for 3 hours in toluene, resulting in a core-shell microstructure. 


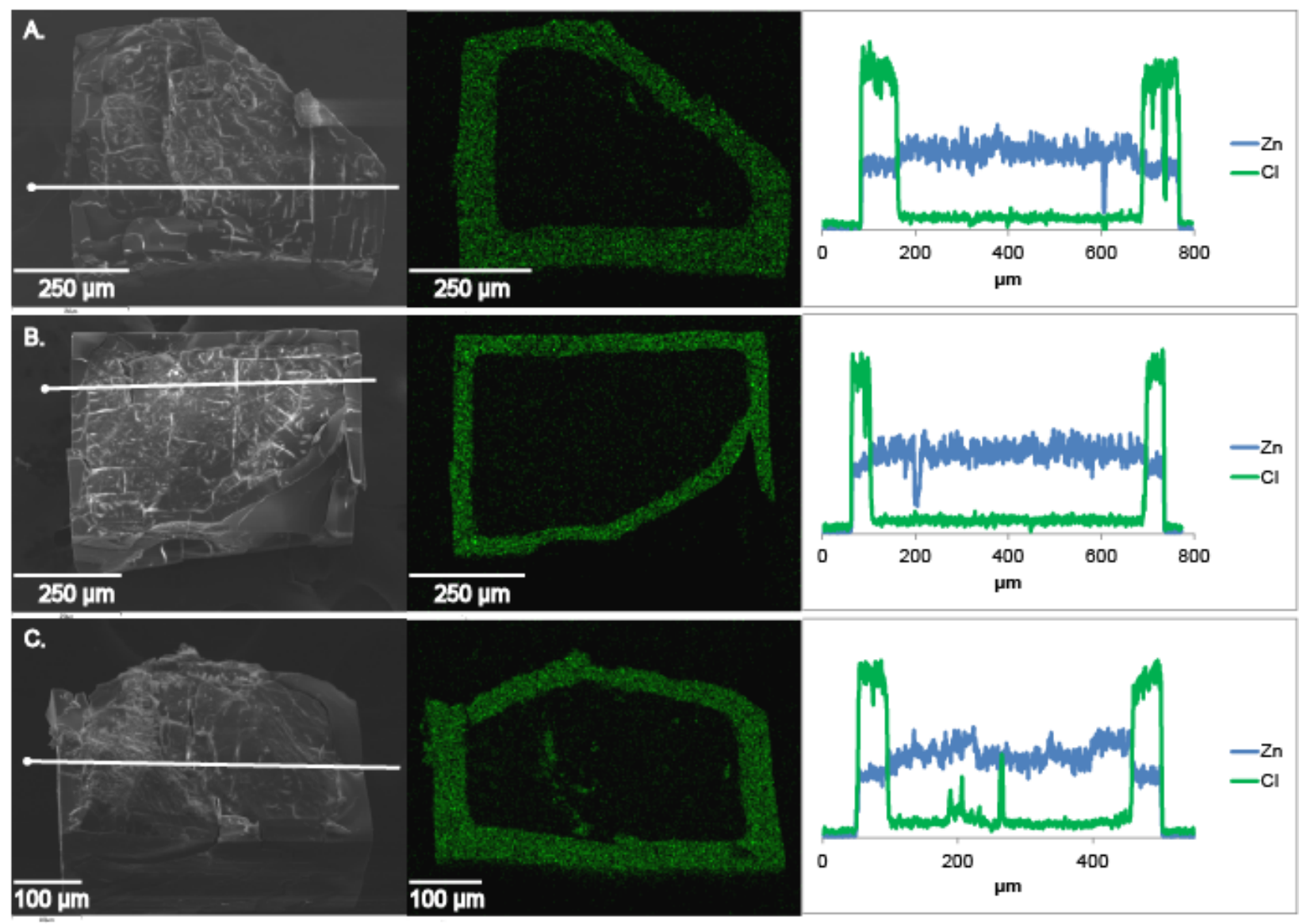

Figure S10. SEM images, EDS maps, and EDS linescans for three trials of PSM on IRMOF-3 with chloroacetyl isocyanate for 6 hours in toluene, resulting in a core-shell microstructure. 


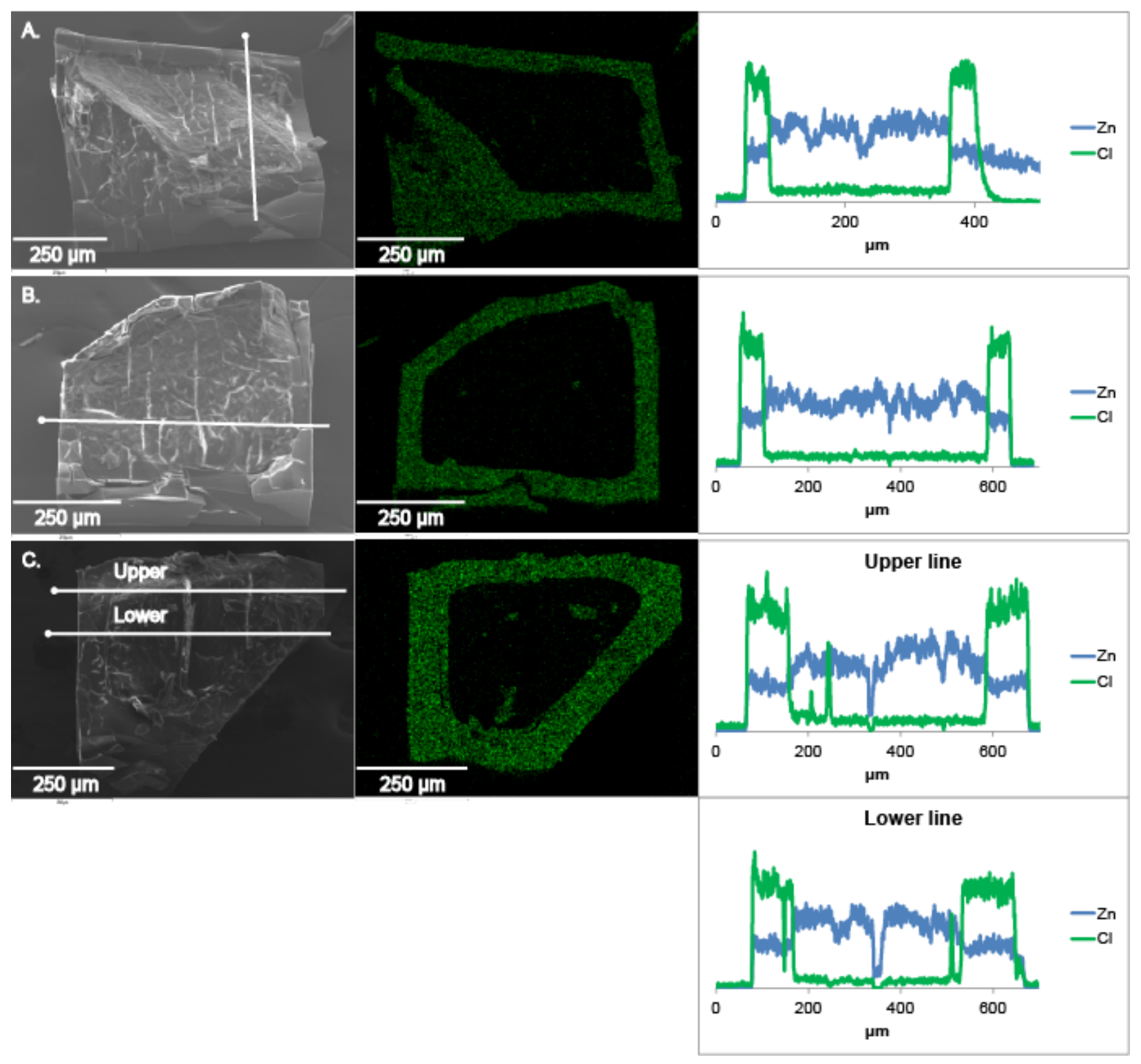

Figure S11. SEM images, EDS maps, and EDS linescans for three trials of PSM on IRMOF-3 with chloroacetyl isocyanate for 9 hours in toluene, resulting in a core-shell microstructure. Trial C has two linescans to ensure accurate depictions of the depth of functionalization with two measured regions that are perpendicular to the edge of the MOF. 


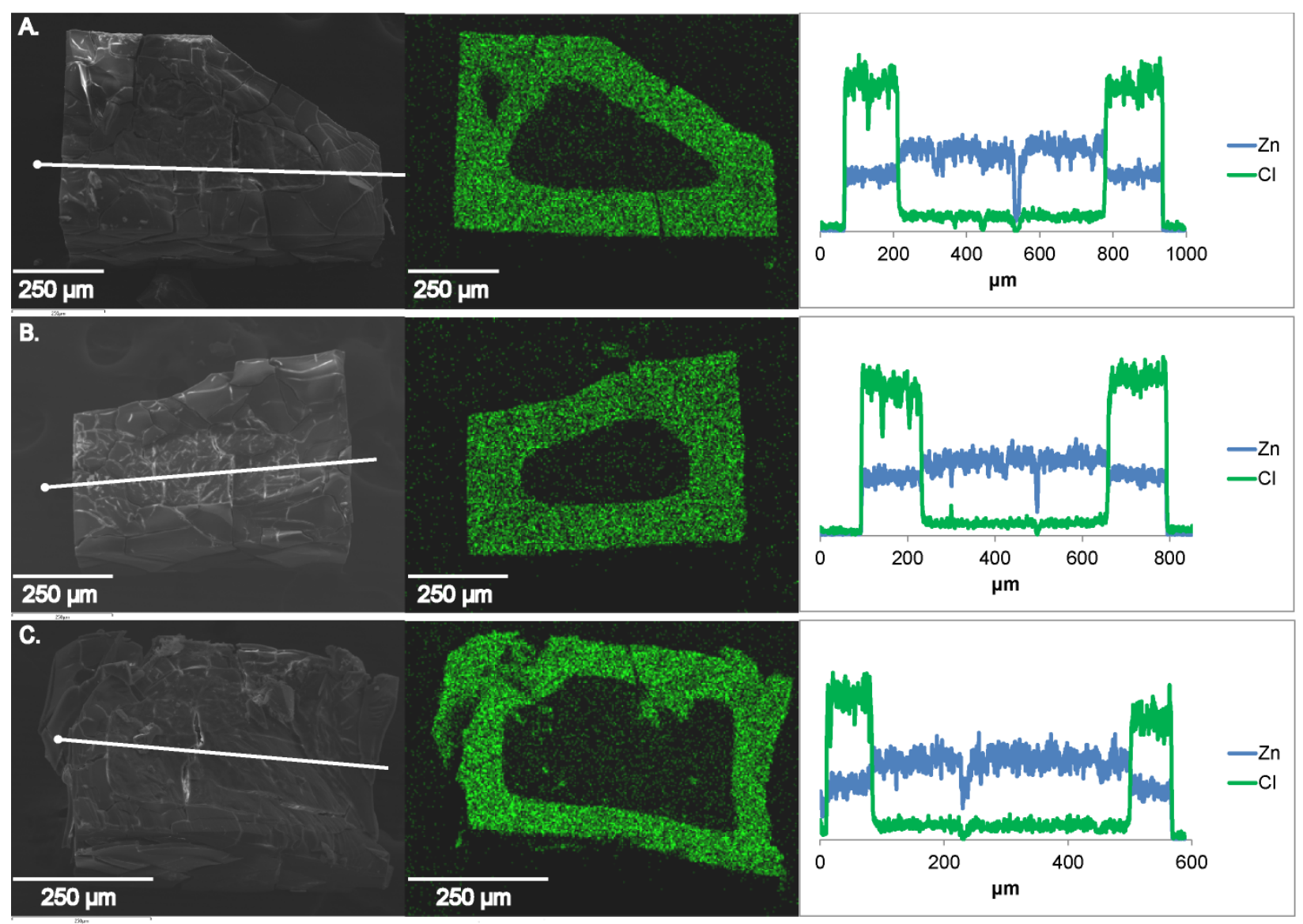

Figure S12. SEM images, EDS maps, and EDS linescans for three trials of PSM on IRMOF-3 with chloroacetyl isocyanate for 24 hours in toluene, resulting in a core-shell microstructure. 

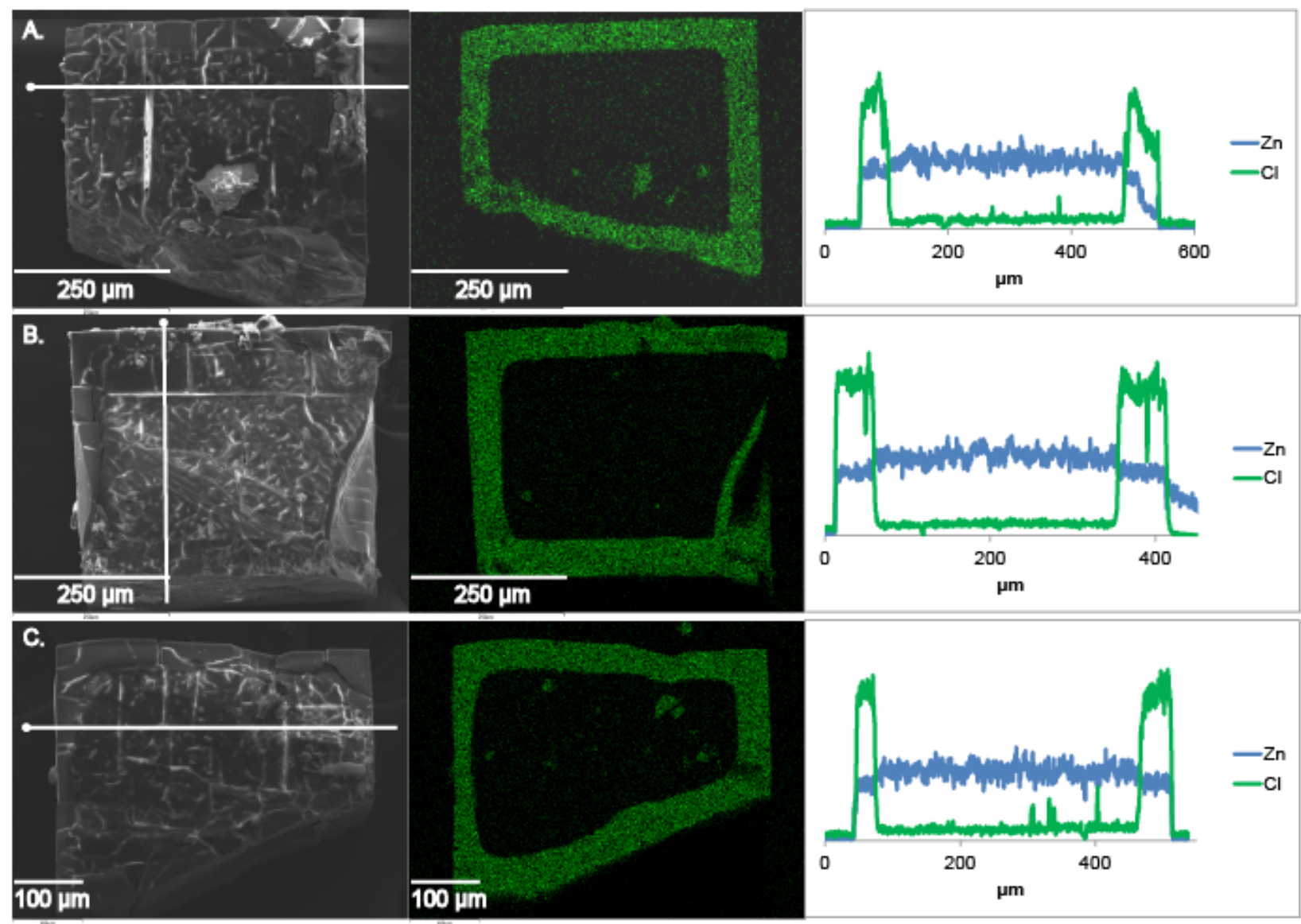

Figure S13. SEM images, EDS maps, and EDS linescans for three trials of PSM on IRMOF-3 with chloroacetyl isocyanate for 3 hours in chloroform, resulting in a core-shell microstructure. 


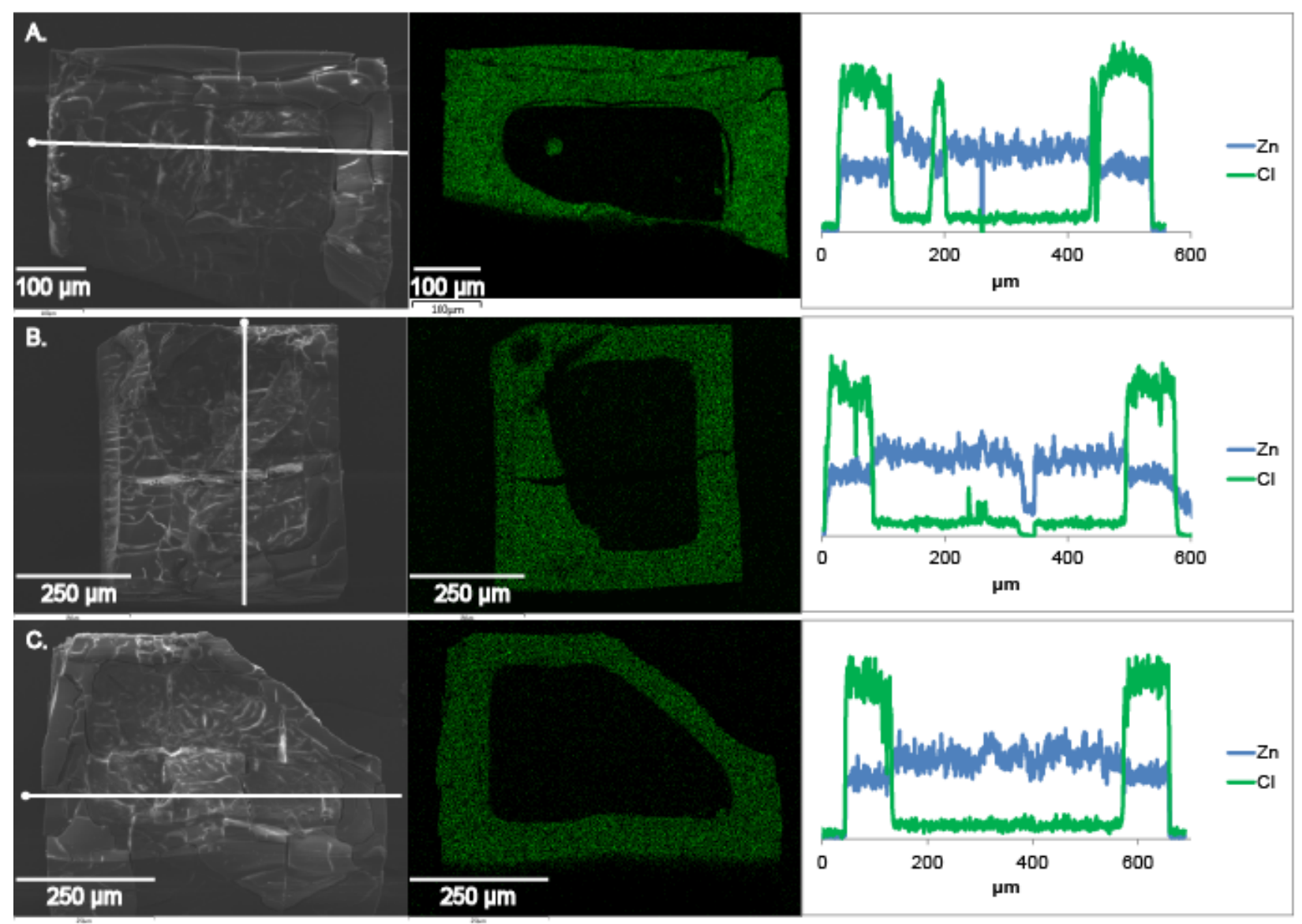

Figure S14. SEM images, EDS maps, and EDS linescans for three trials of PSM on IRMOF-3 with chloroacetyl isocyanate for 6 hours in chloroform, resulting in a core-shell microstructure. 


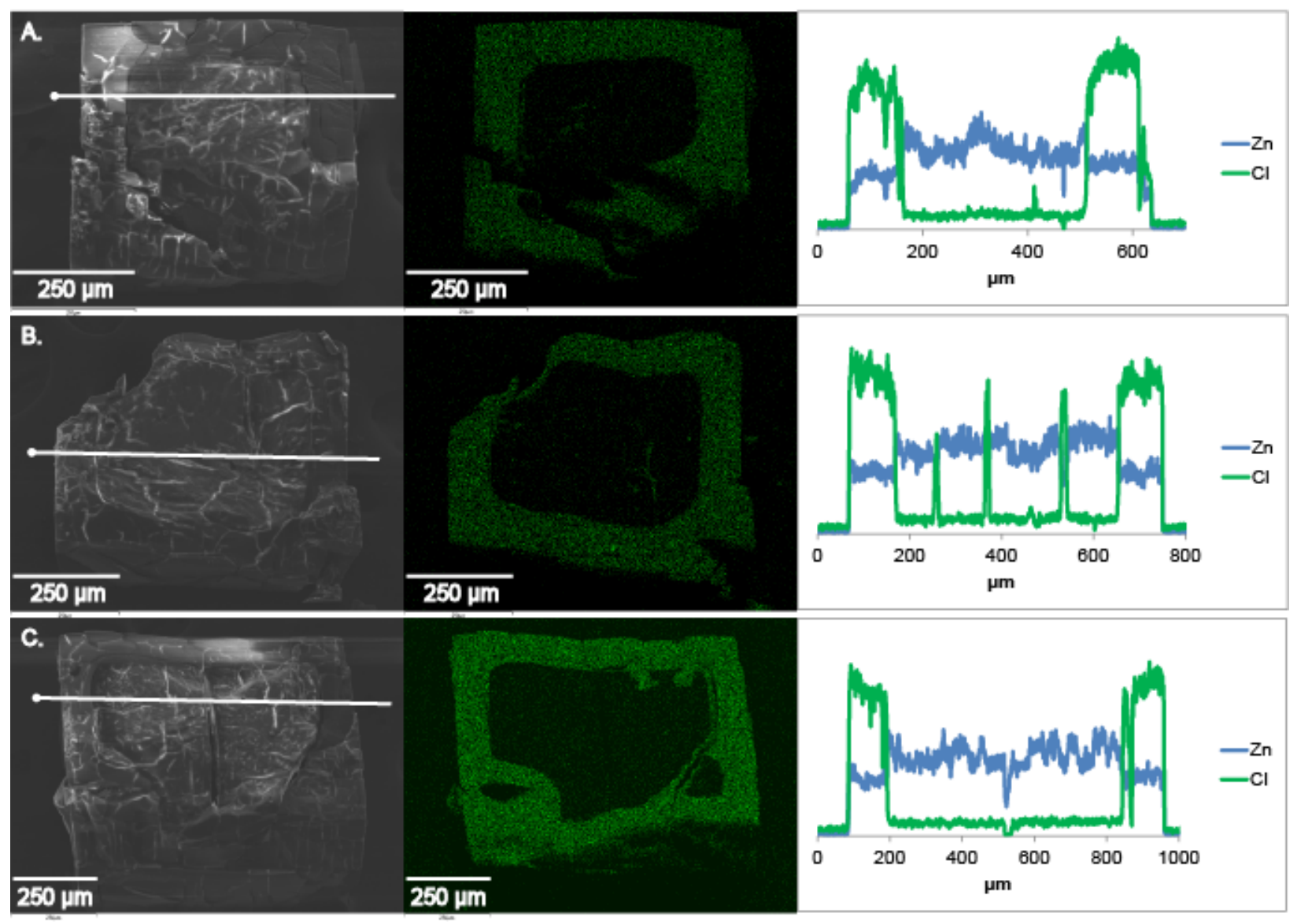

Figure S15. SEM images, EDS maps, and EDS linescans for three trials of PSM on IRMOF-3 with chloroacetyl isocyanate for 9 hours in chloroform, resulting in a core-shell microstructure. 


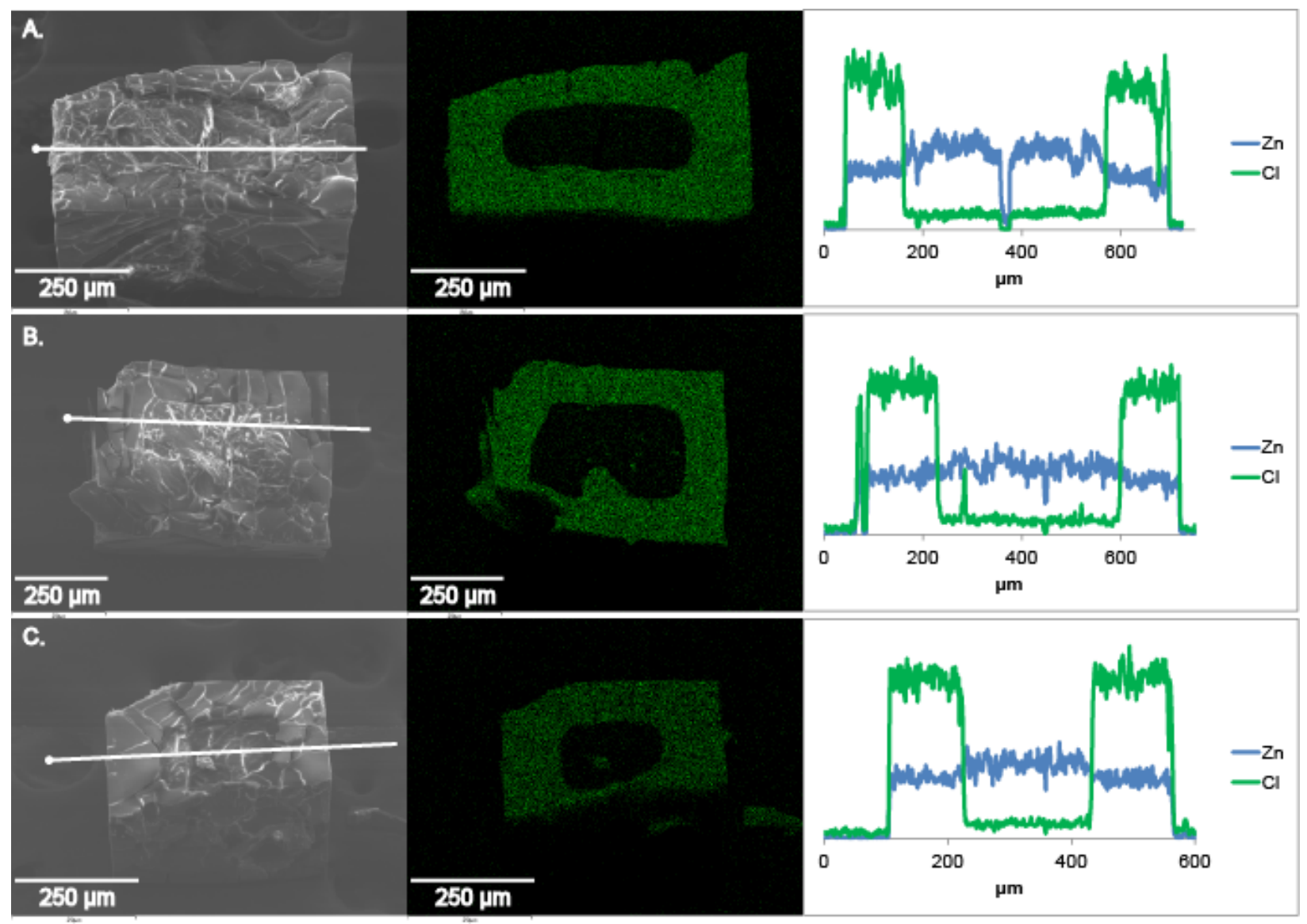

Figure S16. SEM images, EDS maps, and EDS linescans for three trials of PSM on IRMOF-3 with chloroacetyl isocyanate for 24 hours in chloroform, resulting in a core-shell microstructure. 


\section{Experimental IR Kinetics Data}

\section{Reaction of chloroacetyl isocyanate and butyl anthranilate, $40 \mathrm{mM}$ each:}

IR spectra show that after mixing chloroacetyl isocyanate with butyl anthranilate, the isocyanate peak at $2252.5 \mathrm{~cm}^{-1}$ in toluene and $2254.4 \mathrm{~cm}^{-1}$ in chloroform disappears immediately. The urethane product peak at $1531.2 \mathrm{~cm}^{-1}$ in toluene and $1533.2 \mathrm{~cm}^{-1}$ in chloroform appears immediately. 

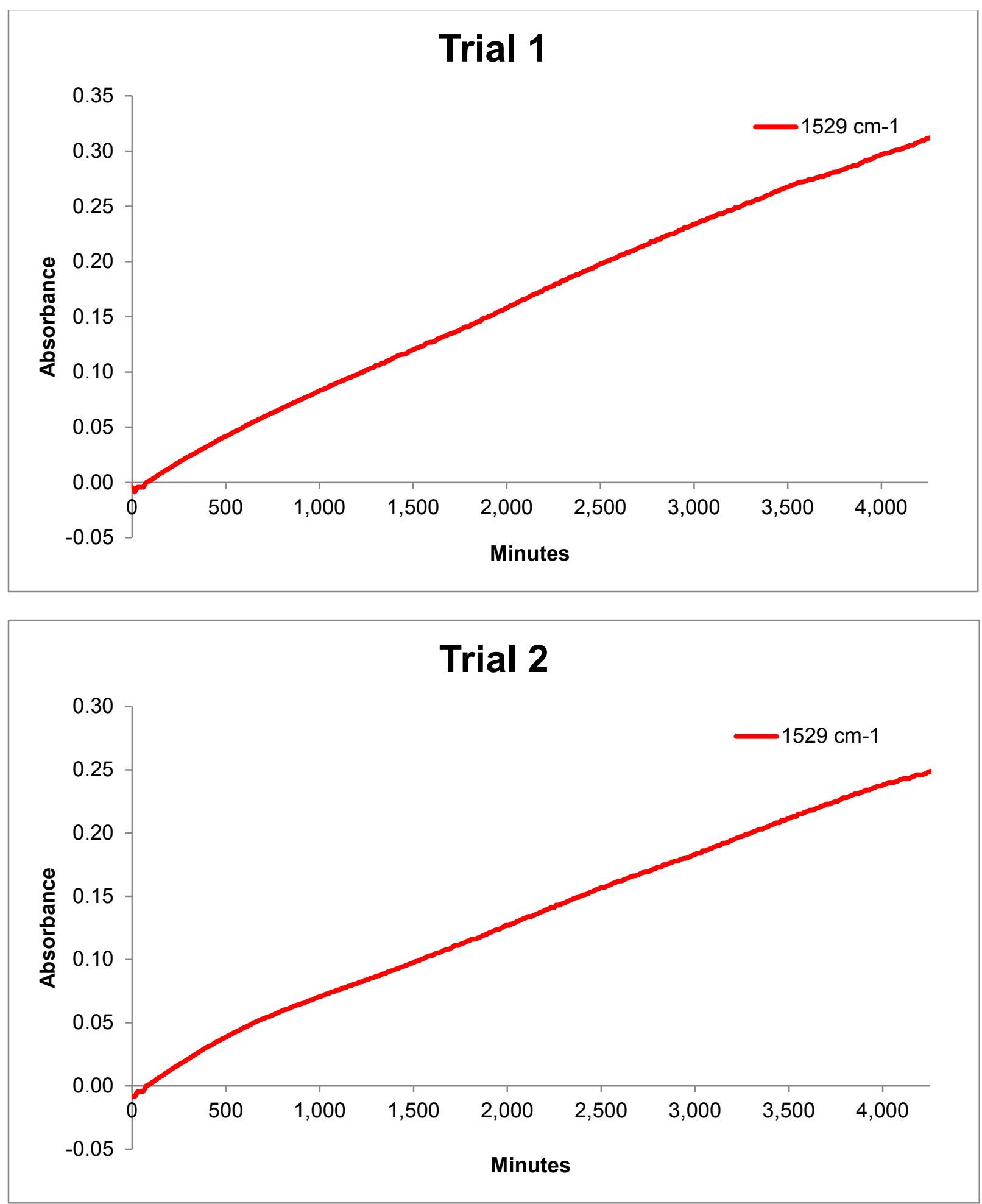

Figure S17. FTIR kinetic measurements for the generation of product, tracked using the peak at $1529 \mathrm{~cm}^{-}$ ${ }^{1}$, for the reaction between butyl anthranilate and 2-chloroethyl isocyanate in toluene, $200 \mathrm{mM}$ each. 

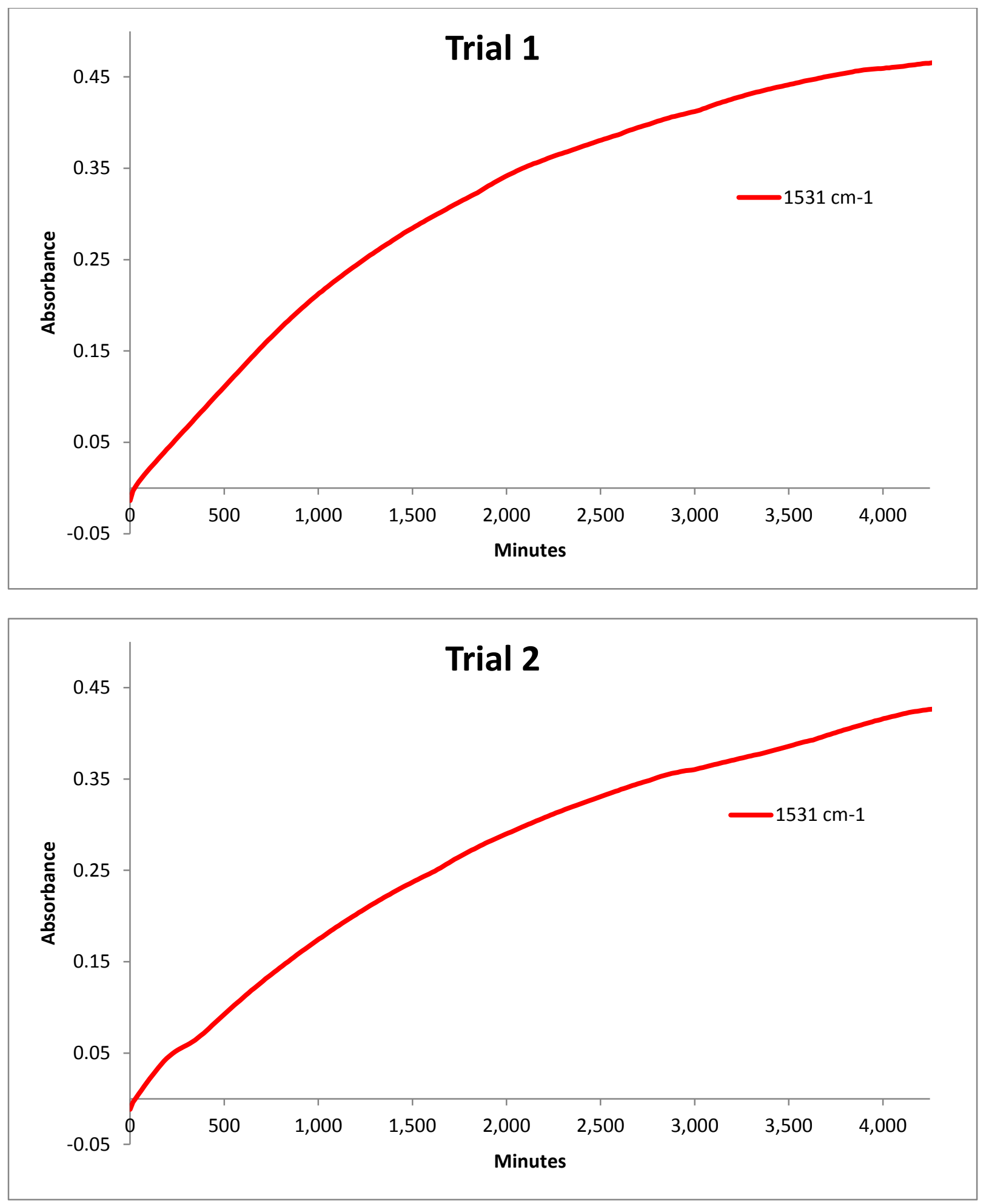

Figure S18. FTIR kinetic measurements for the generation of product, tracked using the peak at $1531 \mathrm{~cm}^{-}$ ${ }^{1}$, for the reaction between butyl anthranilate and 2-chloroethyl isocyanate in chloroform, $200 \mathrm{mM}$ each. 


\section{Experimental ${ }^{1} \mathrm{H}$ NMR Spectroscopy Data}

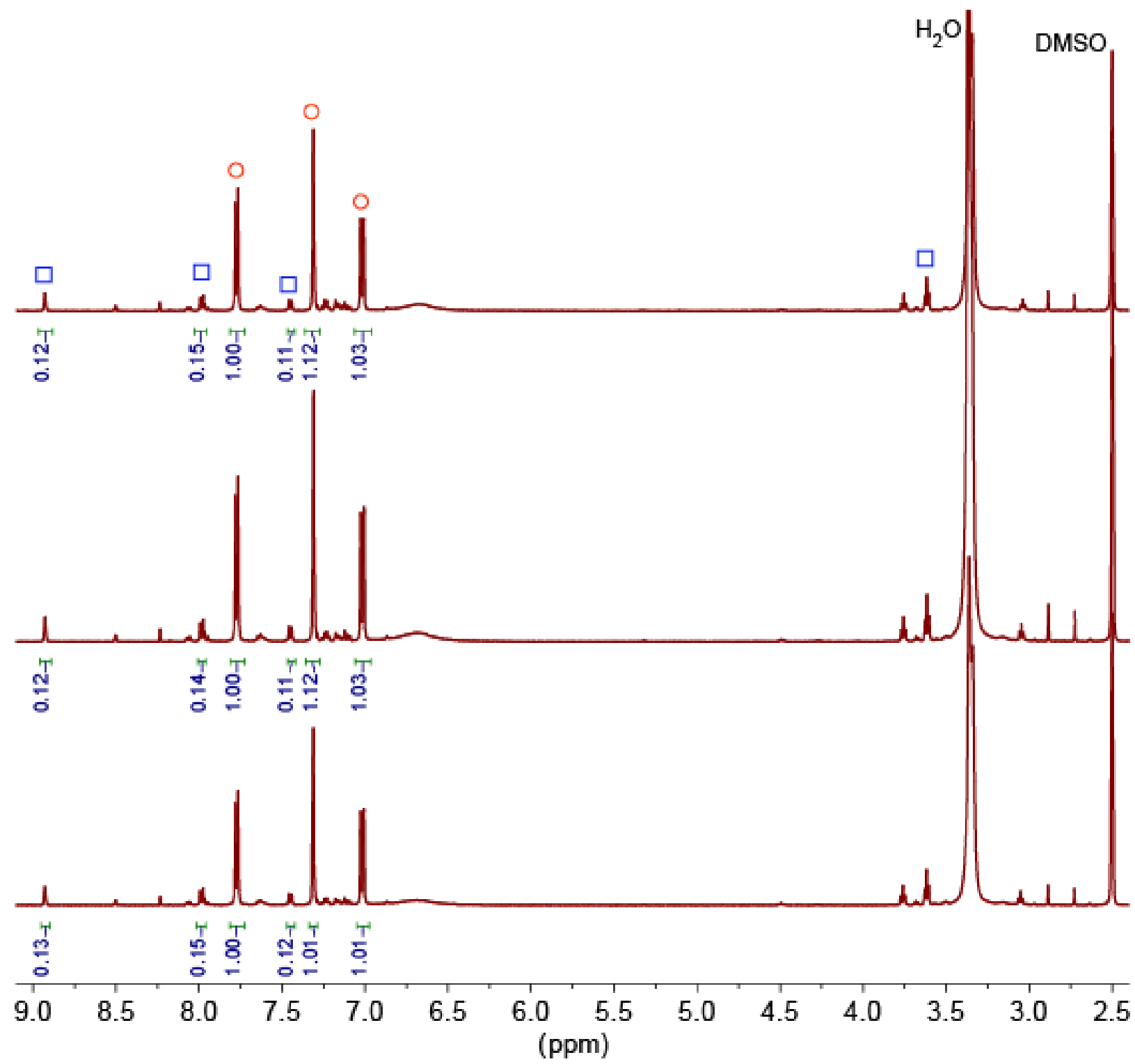

Figure S19. ${ }^{1} \mathrm{H}$ NMR spectra for three trials of digested IRMOF-3 modified with 2-chloroethyl isocyanate in toluene for $3 \mathrm{~h}$. Red circles mark peaks related to unmodified IRMOF-3, and blue squares mark peaks related to IRMOF-3 after PSM with 2-chloroethyl isocyanate. Integration values for the determination of conversion percentage are taken from peaks at $7.78 \mathrm{ppm}$ for IRMOF-3 and $7.45 \mathrm{ppm}$ for IRMOF-3 after PSM. 


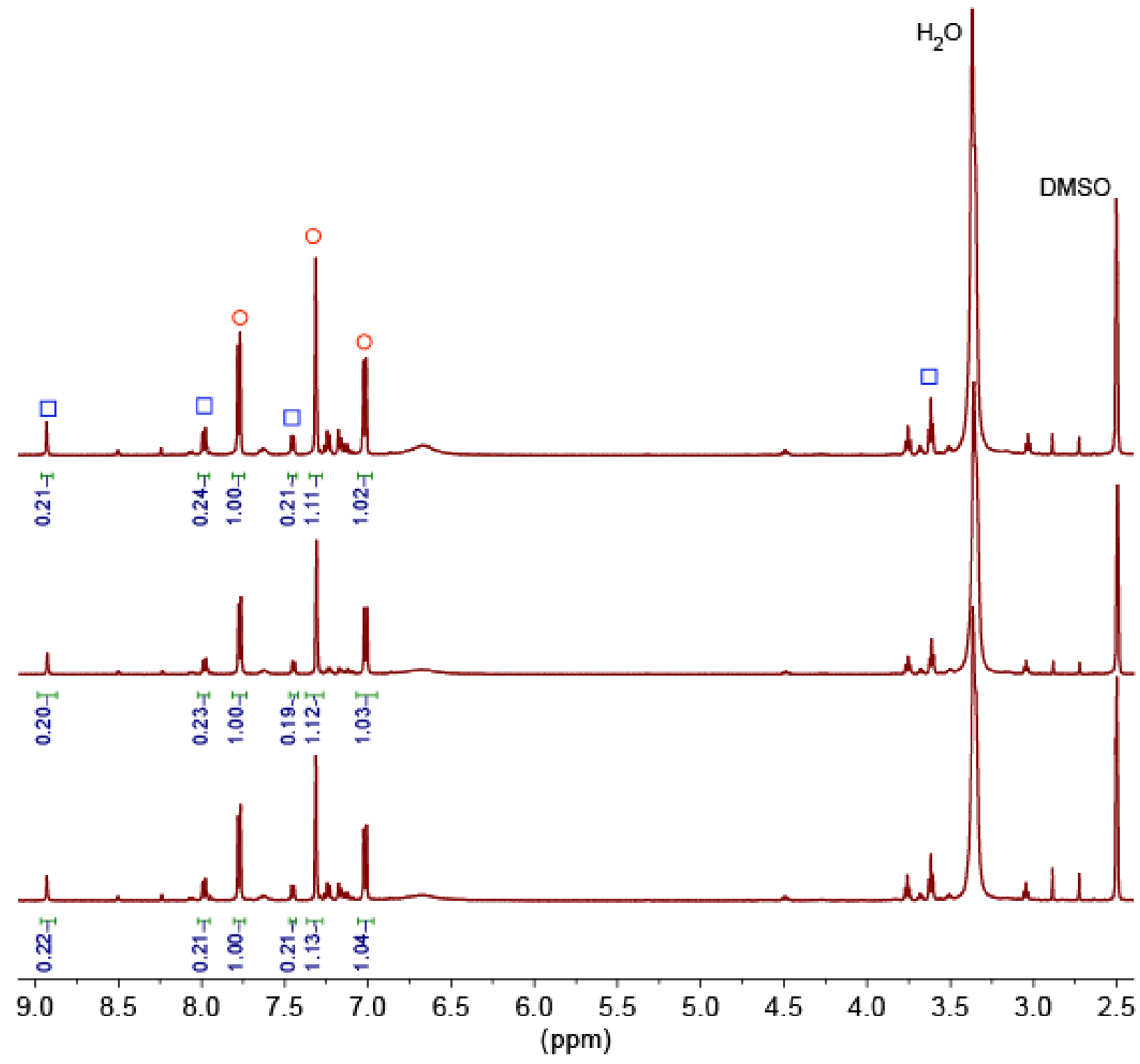

Figure S20. ${ }^{1} \mathrm{H}$ NMR spectra for three trials of digested IRMOF-3 modified with 2-chloroethyl isocyanate in toluene for 6 h. Red circles mark peaks related to unmodified IRMOF-3, and blue squares mark peaks related to IRMOF-3 after PSM with 2-chloroethyl isocyanate. Integration values for the determination of conversion percentage are taken from peaks at $7.78 \mathrm{ppm}$ for IRMOF-3 and $7.45 \mathrm{ppm}$ for IRMOF-3 after PSM. 


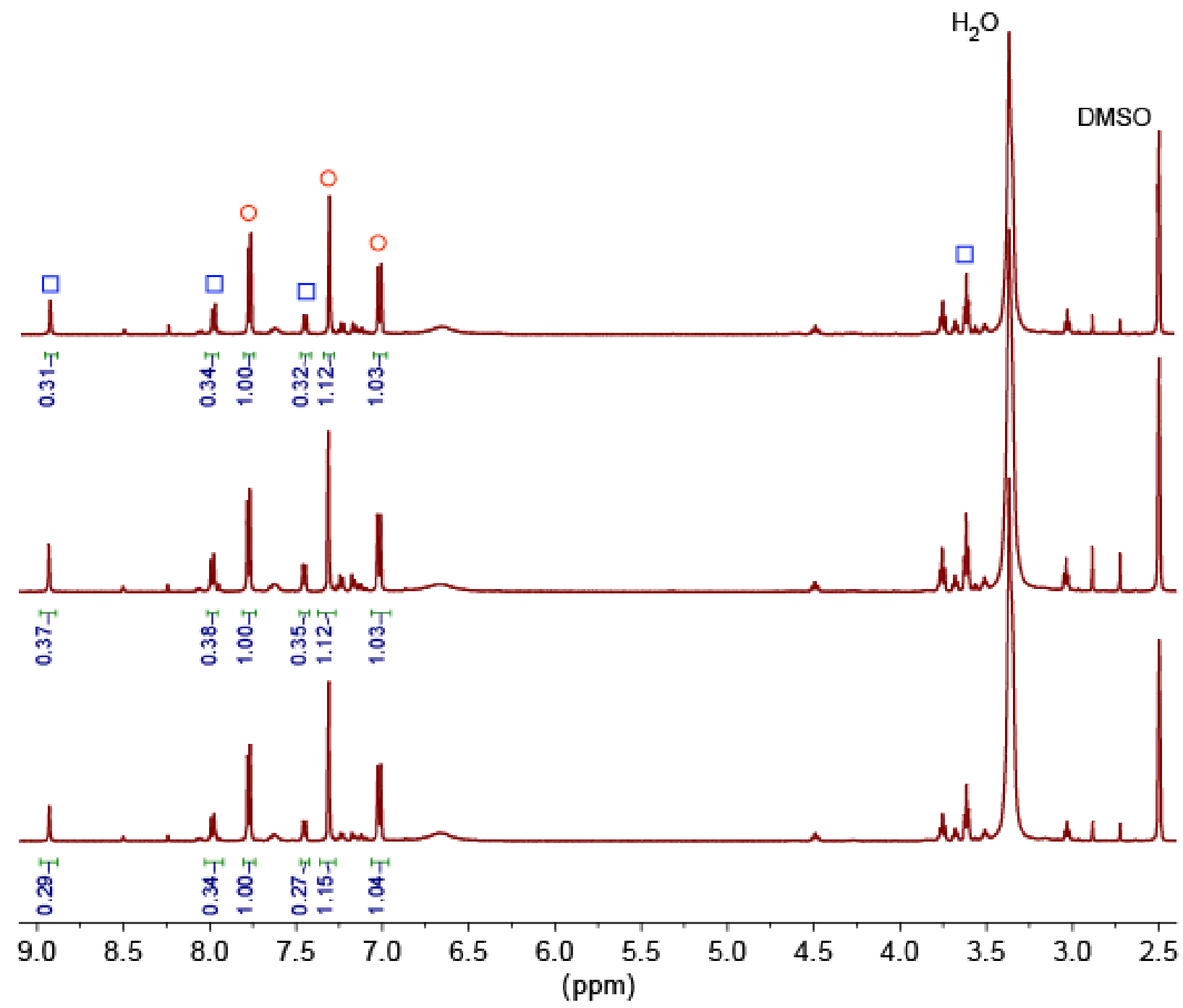

Figure S21. ${ }^{1} \mathrm{H}$ NMR spectra for three trials of digested IRMOF-3 modified with 2-chloroethyl isocyanate in toluene for $9 \mathrm{~h}$. Red circles mark peaks related to unmodified IRMOF-3, and blue squares mark peaks related to IRMOF-3 after PSM with 2-chloroethyl isocyanate. Integration values for the determination of conversion percentage are taken from peaks at $7.78 \mathrm{ppm}$ for IRMOF-3 and $7.45 \mathrm{ppm}$ for IRMOF-3 after PSM. 


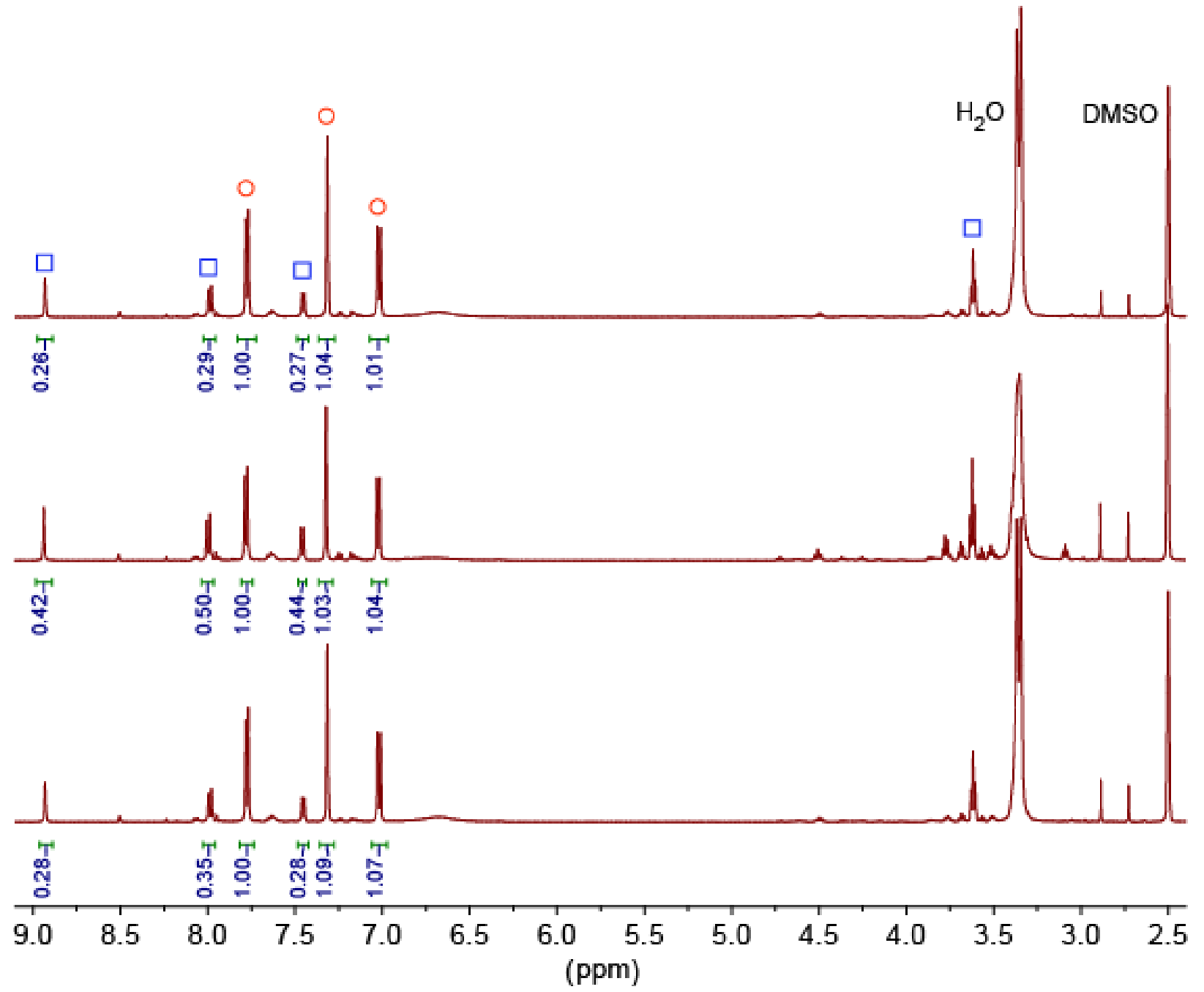

Figure S22. ${ }^{1} \mathrm{H}$ NMR spectra for three trials of digested IRMOF-3 modified with 2-chloroethyl isocyanate in toluene for $24 \mathrm{~h}$. Red circles mark peaks related to unmodified IRMOF-3, and blue squares mark peaks related to IRMOF-3 after PSM with 2-chloroethyl isocyanate. Integration values for the determination of conversion percentage are taken from peaks at $7.78 \mathrm{ppm}$ for IRMOF-3 and $7.45 \mathrm{ppm}$ for IRMOF-3 after PSM. 


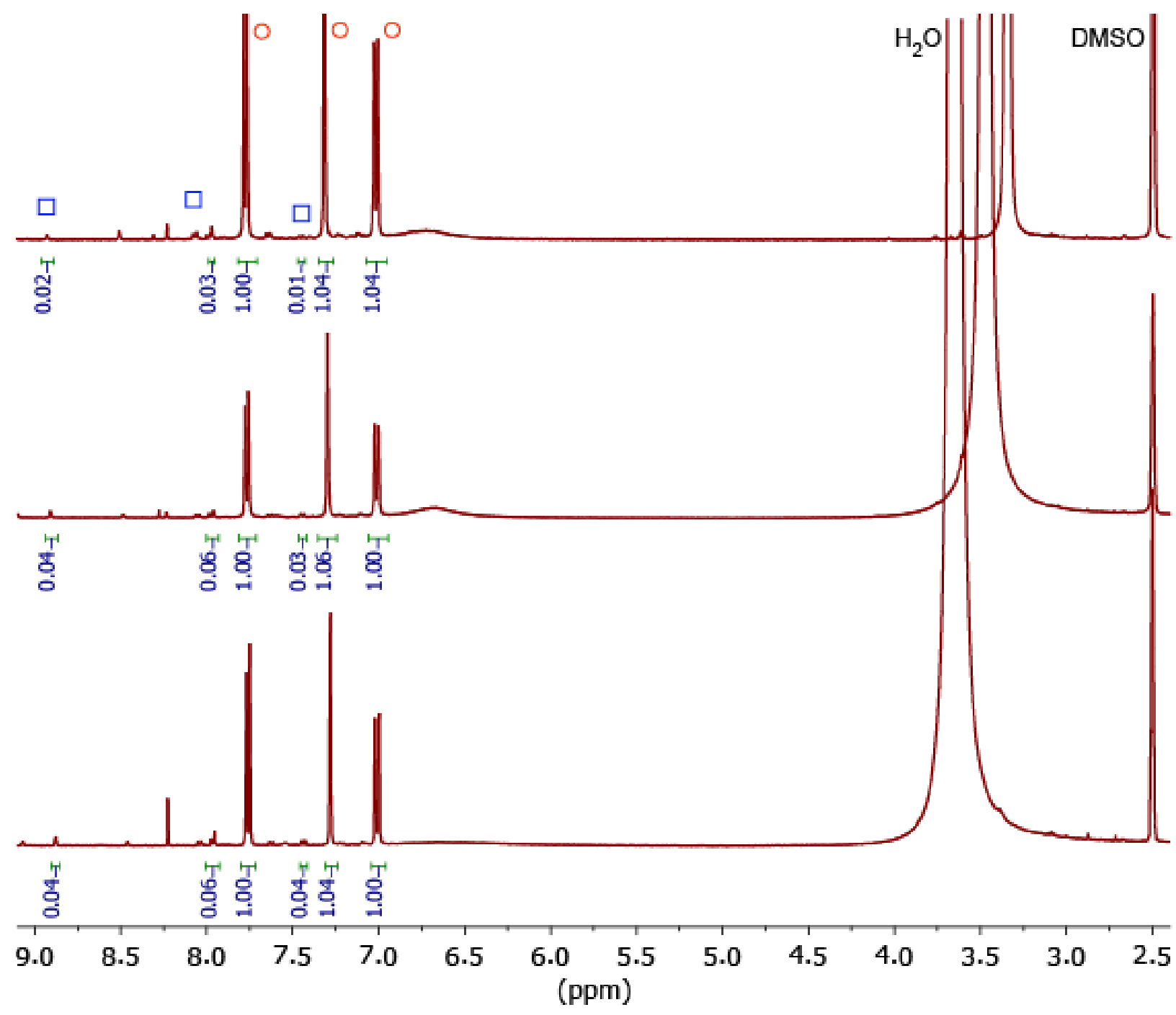

Figure S23. ${ }^{1} \mathrm{H}$ NMR spectra for three trials of digested IRMOF-3 modified with 2-chloroethyl isocyanate in chloroform for $3 \mathrm{~h}$. Red circles mark peaks related to unmodified IRMOF-3, and blue squares mark peaks related to IRMOF-3 after PSM with 2-chloroethyl isocyanate. Integration values for the determination of conversion percentage are taken from peaks at $7.78 \mathrm{ppm}$ for IRMOF-3 and $7.45 \mathrm{ppm}$ for IRMOF-3 after PSM. 


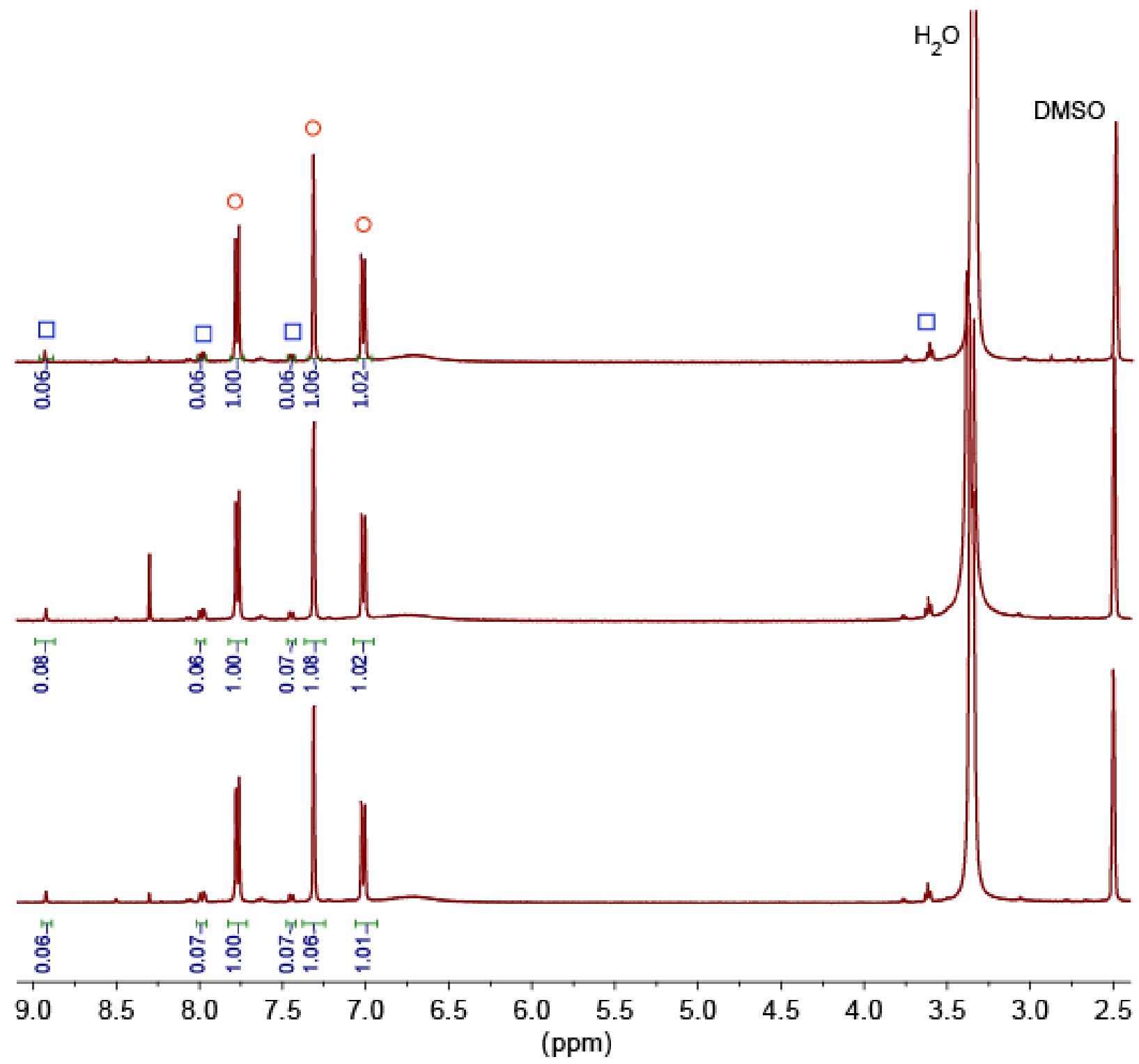

Figure S24. ${ }^{1} \mathrm{H}$ NMR spectra for three trials of digested IRMOF-3 modified with 2-chloroethyl isocyanate in chloroform for $6 \mathrm{~h}$. Red circles mark peaks related to unmodified IRMOF-3, and blue squares mark peaks related to IRMOF-3 after PSM with 2-chloroethyl isocyanate. Integration values for the determination of conversion percentage are taken from peaks at $7.78 \mathrm{ppm}$ for IRMOF-3 and $7.45 \mathrm{ppm}$ for IRMOF-3 after PSM. 


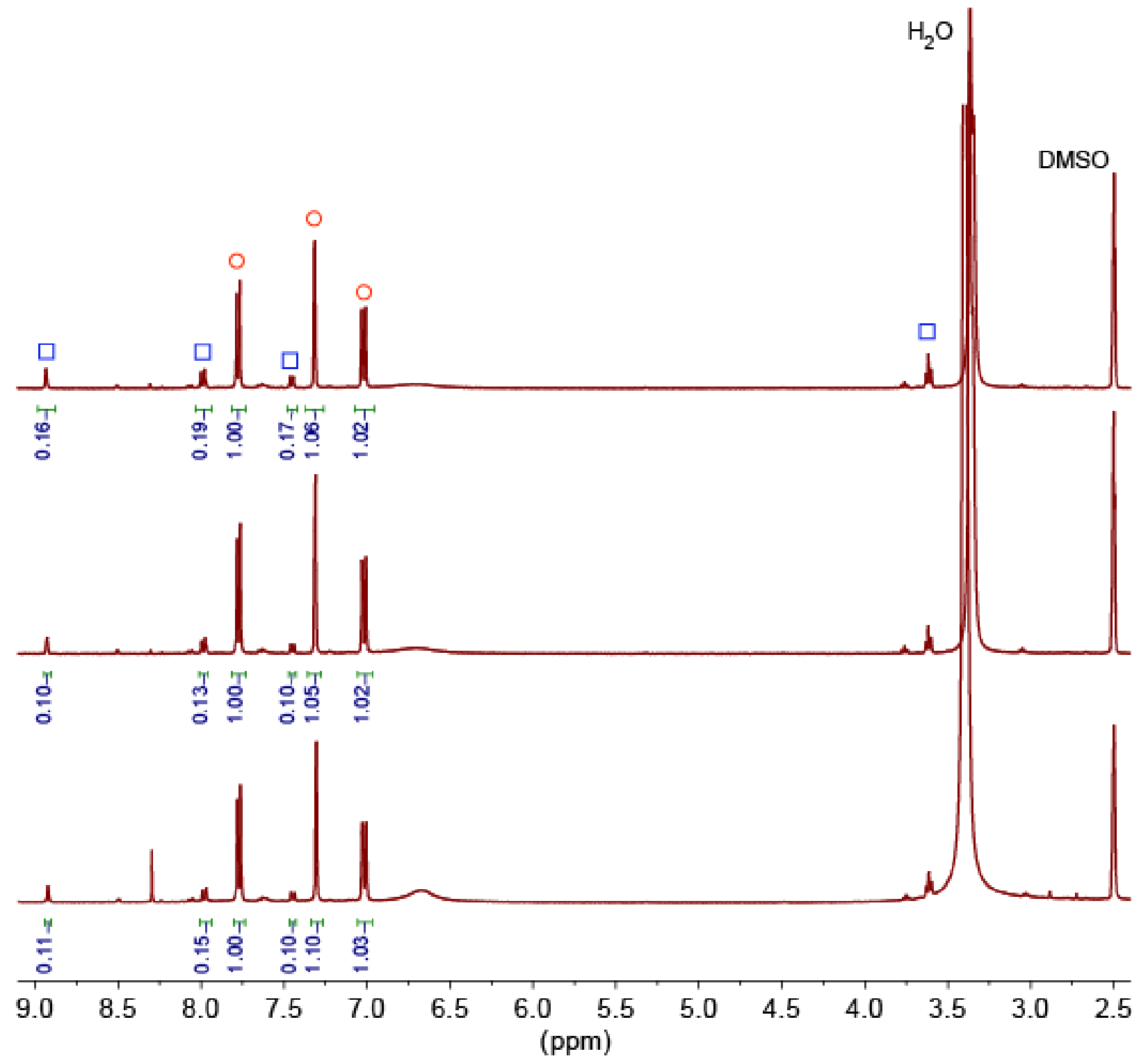

Figure S25. ${ }^{1} \mathrm{H}$ NMR spectra for three trials of digested IRMOF-3 modified with 2-chloroethyl isocyanate in chloroform for $9 \mathrm{~h}$. Red circles mark peaks related to unmodified IRMOF-3, and blue squares mark peaks related to IRMOF-3 after PSM with 2-chloroethyl isocyanate. Integration values for the determination of conversion percentage are taken from peaks at $7.78 \mathrm{ppm}$ for IRMOF-3 and $7.45 \mathrm{ppm}$ for IRMOF-3 after PSM. 


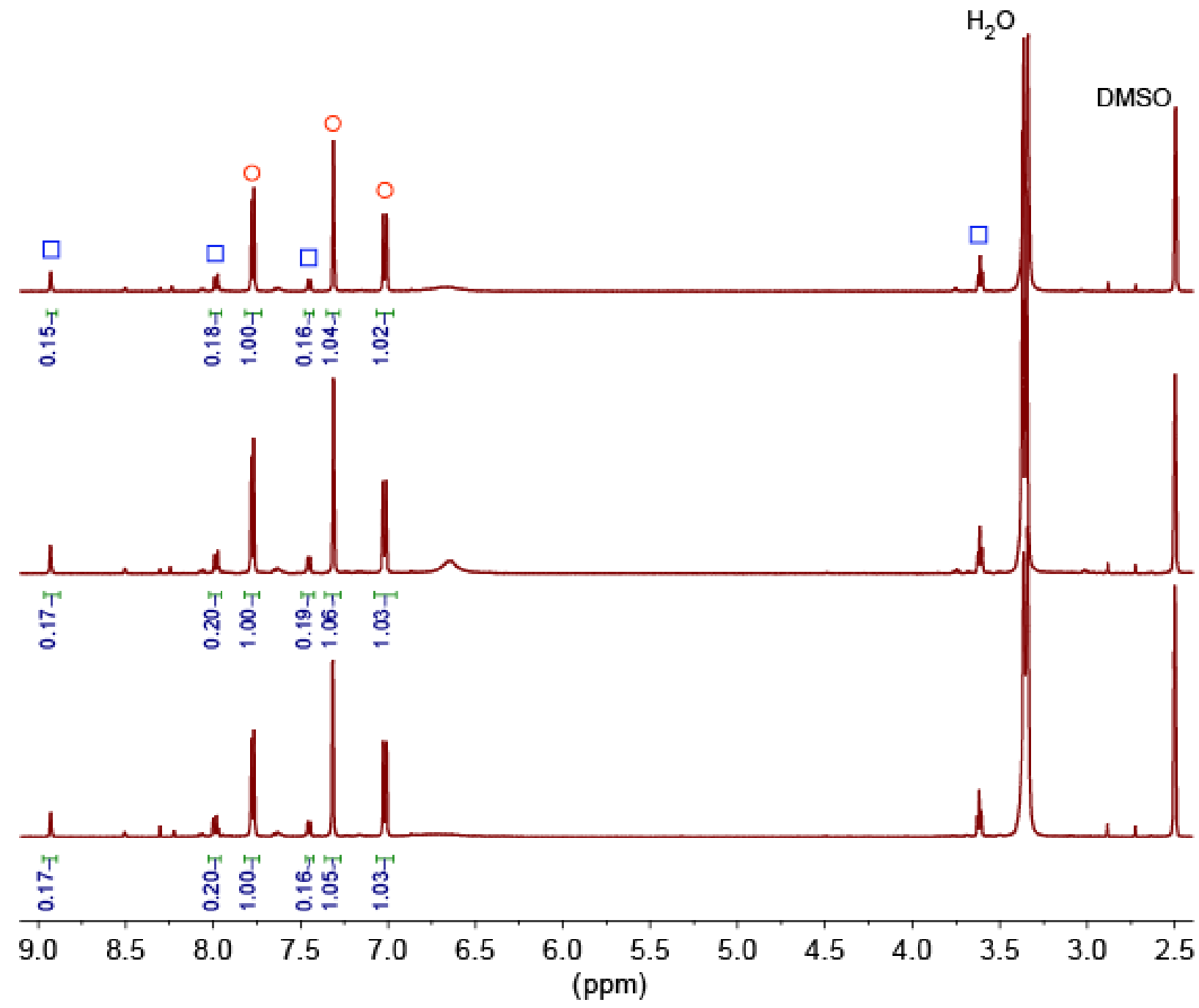

Figure S26. ${ }^{1} \mathrm{H}$ NMR spectra for three trials of digested IRMOF-3 modified with 2-chloroethyl isocyanate in chloroform for $24 \mathrm{~h}$. Red circles mark peaks related to unmodified IRMOF-3, and blue squares mark peaks related to IRMOF-3 after PSM with 2-chloroethyl isocyanate. Integration values for the determination of conversion percentage are taken from peaks at $7.78 \mathrm{ppm}$ for IRMOF-3 and $7.45 \mathrm{ppm}$ for IRMOF-3 after PSM. 


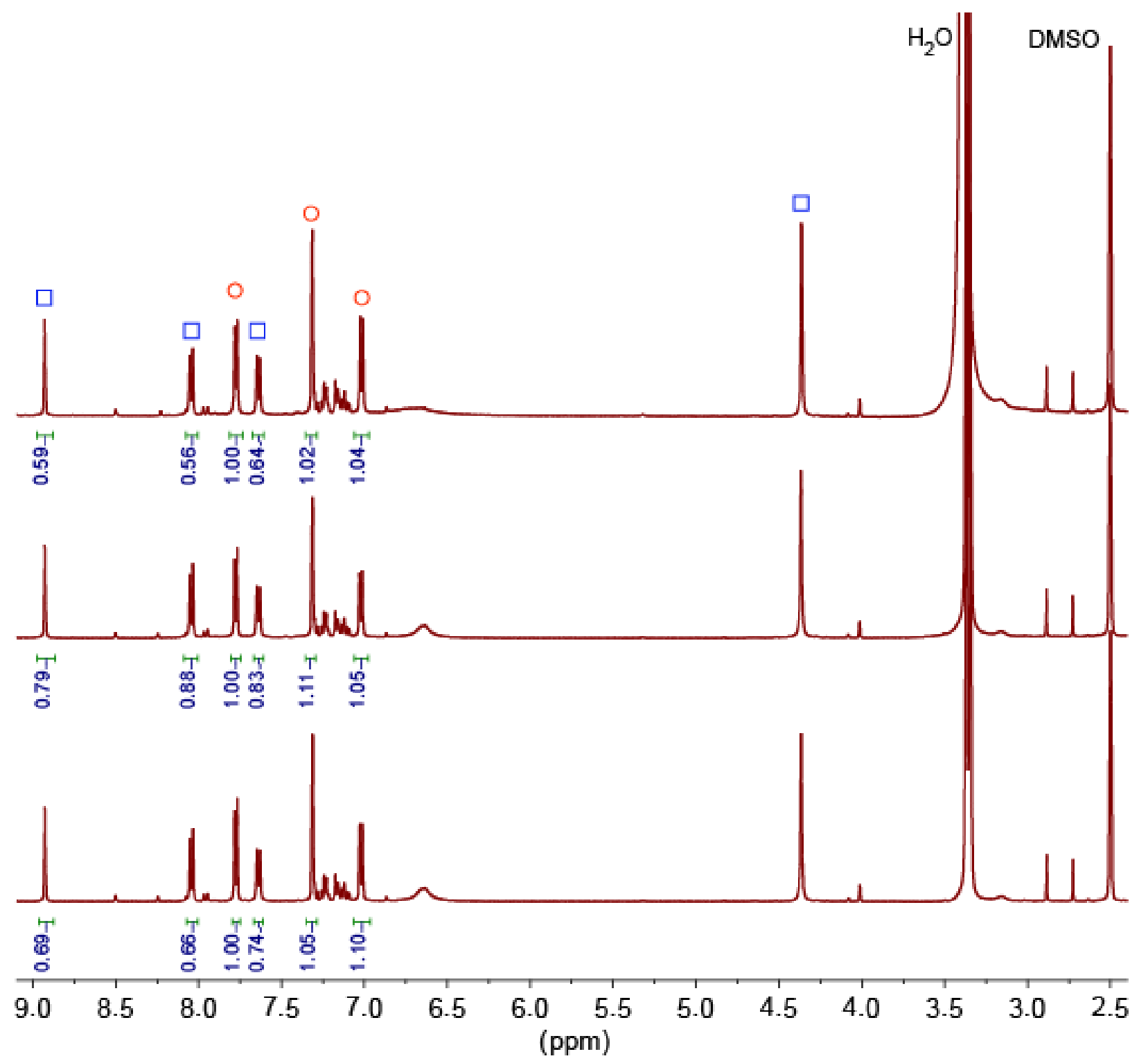

Figure S27. ${ }^{1} \mathrm{H}$ NMR spectra for three trials of digested IRMOF-3 modified with chloroacetyl isocyanate in toluene for $3 \mathrm{~h}$. Red circles mark peaks related to unmodified IRMOF-3, and blue squares mark peaks related to IRMOF-3 after PSM with chloroacetyl isocyanate. Integration values for the determination of conversion percentage are taken from peaks at $7.78 \mathrm{ppm}$ for IRMOF-3 and $7.65 \mathrm{ppm}$ for IRMOF-3 after PSM. 


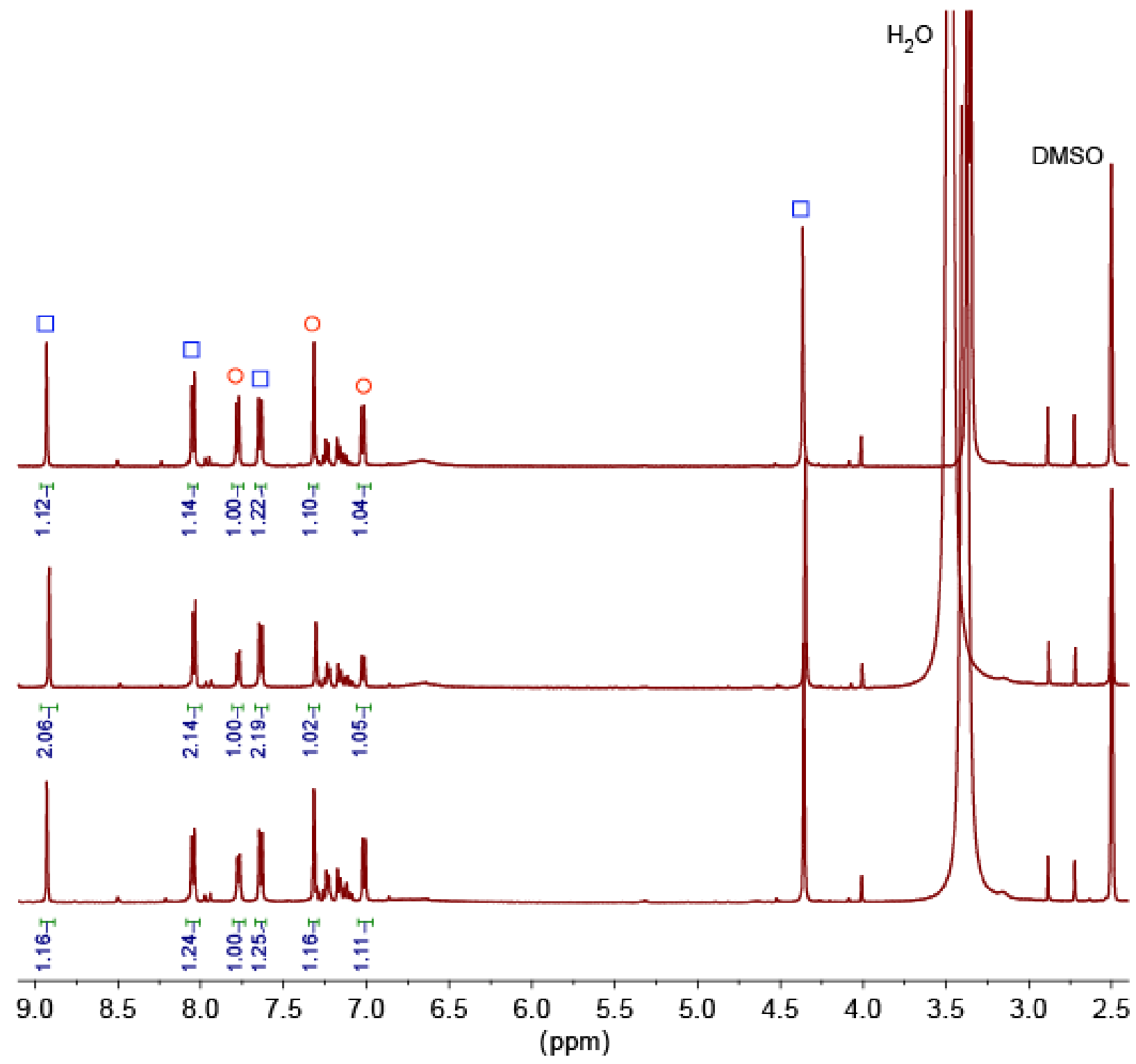

Figure S28. ${ }^{1} \mathrm{H}$ NMR spectra for three trials of digested IRMOF-3 modified with chloroacetyl isocyanate in toluene for $6 \mathrm{~h}$. Red circles mark peaks related to unmodified IRMOF-3, and blue squares mark peaks related to IRMOF-3 after PSM with chloroacetyl isocyanate. Integration values for the determination of conversion percentage are taken from peaks at $7.78 \mathrm{ppm}$ for IRMOF-3 and $7.65 \mathrm{ppm}$ for IRMOF-3 after PSM. 


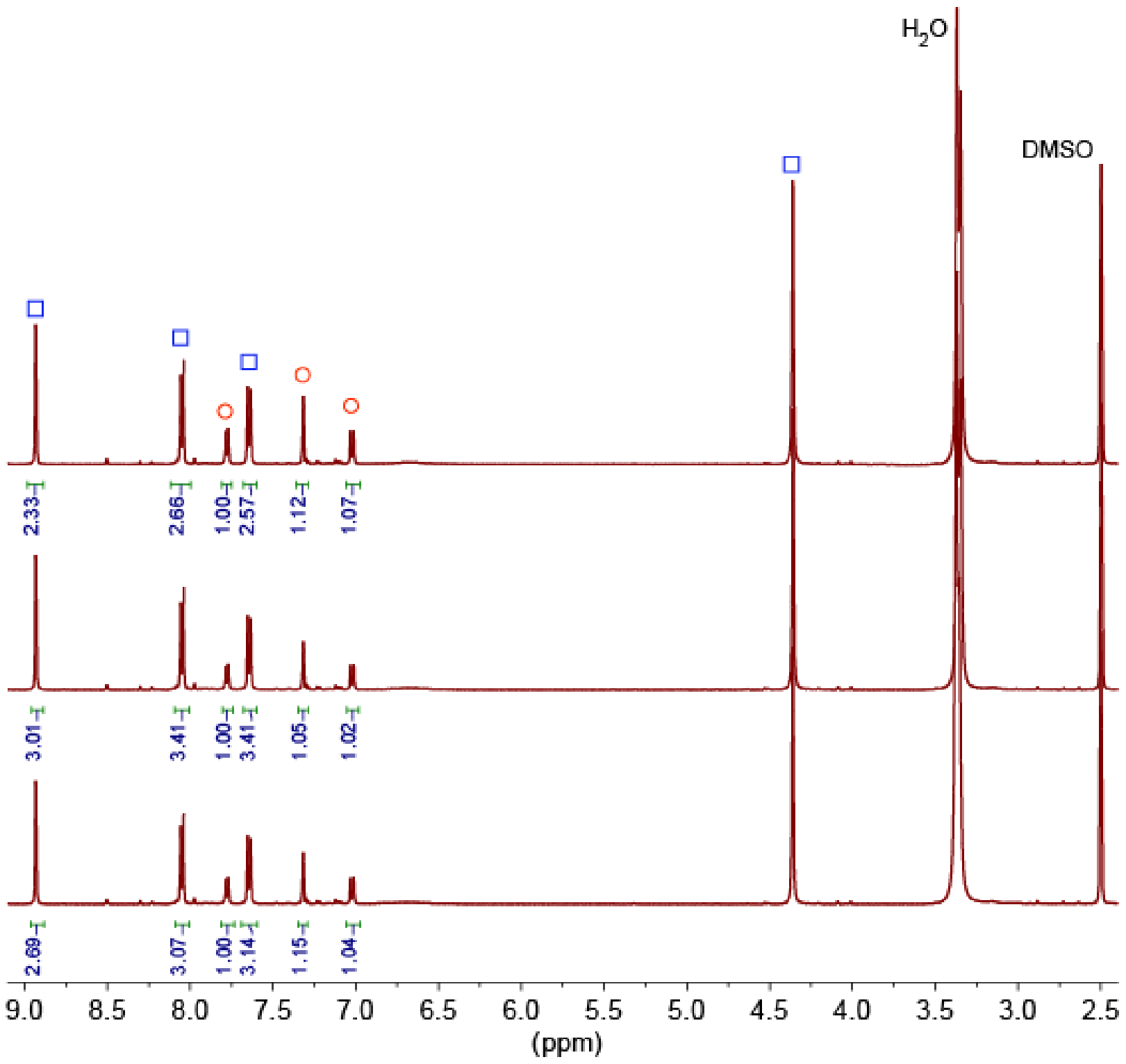

Figure S29. ${ }^{1} \mathrm{H}$ NMR spectra for three trials of digested IRMOF-3 modified with chloroacetyl isocyanate in toluene for $9 \mathrm{~h}$. Red circles mark peaks related to unmodified IRMOF-3, and blue squares mark peaks related to IRMOF-3 after PSM with chloroacetyl isocyanate. Integration values for the determination of conversion percentage are taken from peaks at $7.78 \mathrm{ppm}$ for IRMOF-3 and $7.65 \mathrm{ppm}$ for IRMOF-3 after PSM. 


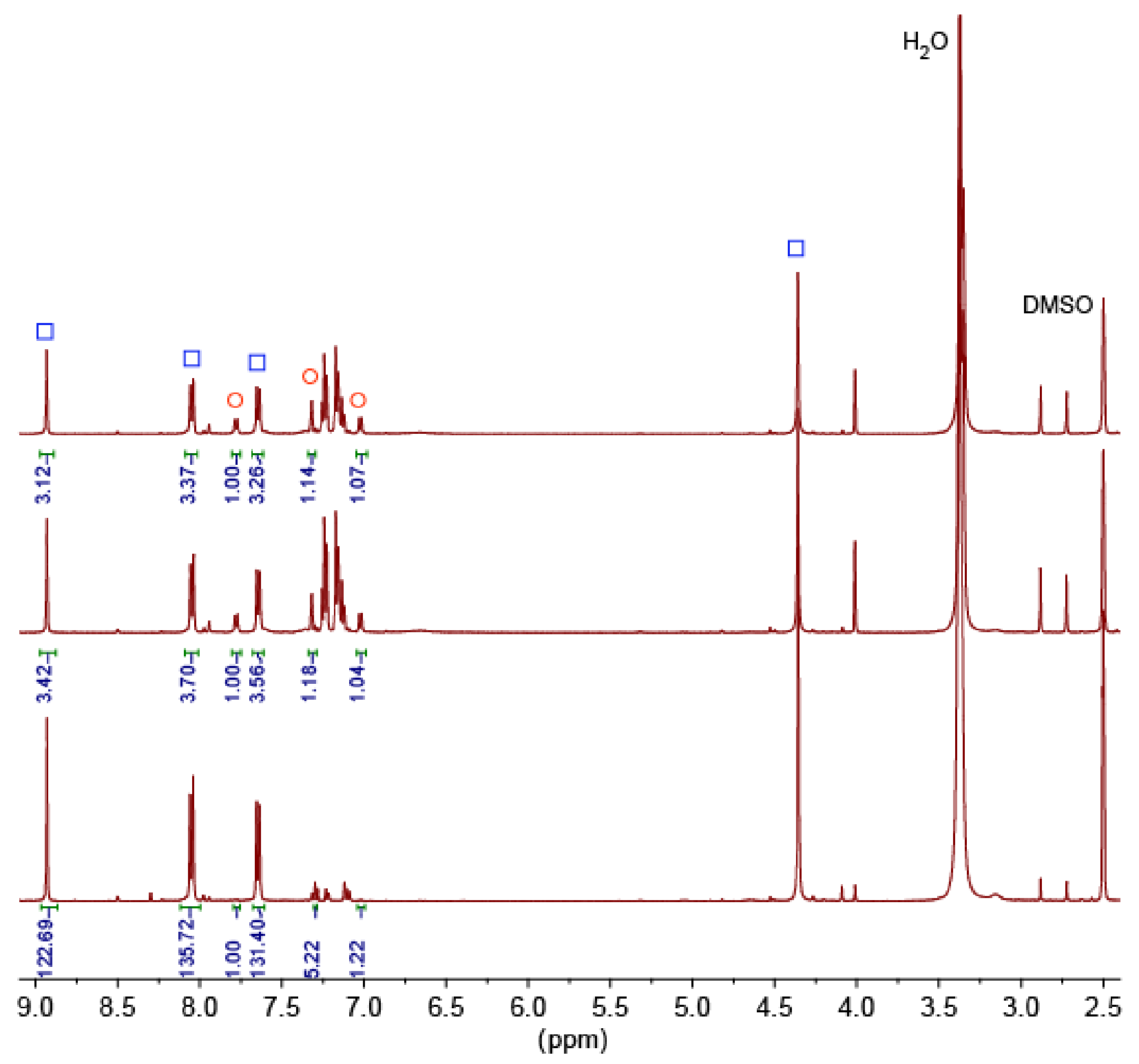

Figure S30. ${ }^{1} \mathrm{H}$ NMR spectra for three trials of digested IRMOF-3 modified with chloroacetyl isocyanate in toluene for $24 \mathrm{~h}$. Red circles mark peaks related to unmodified IRMOF-3, and blue squares mark peaks related to IRMOF-3 after PSM with chloroacetyl isocyanate. Integration values for the determination of conversion percentage are taken from peaks at $7.78 \mathrm{ppm}$ for IRMOF-3 and $7.65 \mathrm{ppm}$ for IRMOF-3 after PSM. 


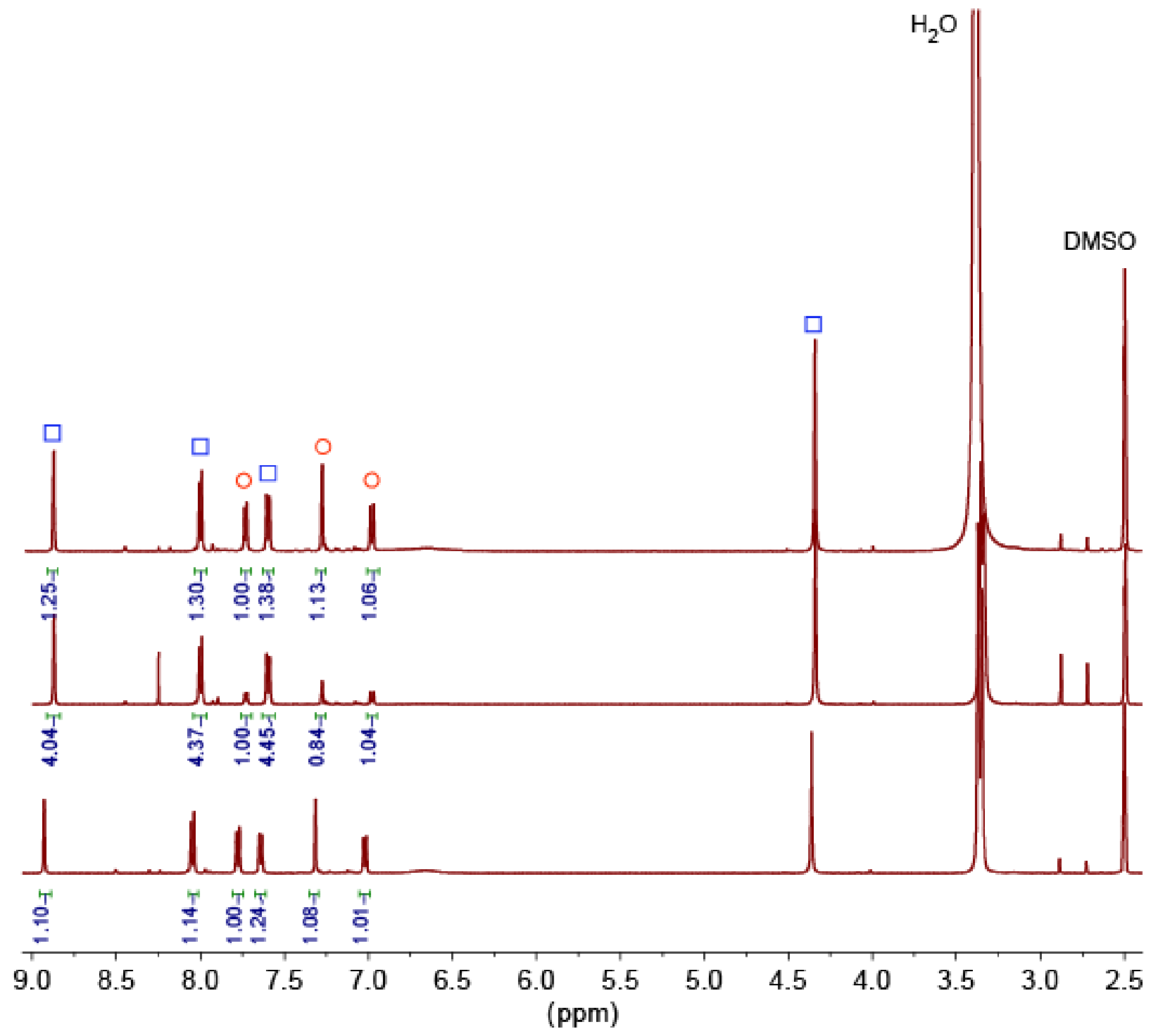

Figure S31. ${ }^{1} \mathrm{H}$ NMR spectra for three trials of digested IRMOF-3 modified with chloroacetyl isocyanate in chloroform for $3 \mathrm{~h}$. Red circles mark peaks related to unmodified IRMOF-3, and blue squares mark peaks related to IRMOF-3 after PSM with chloroacetyl isocyanate. Integration values for the determination of conversion percentage are taken from peaks at $7.78 \mathrm{ppm}$ for IRMOF-3 and $7.65 \mathrm{ppm}$ for IRMOF-3 after PSM. 


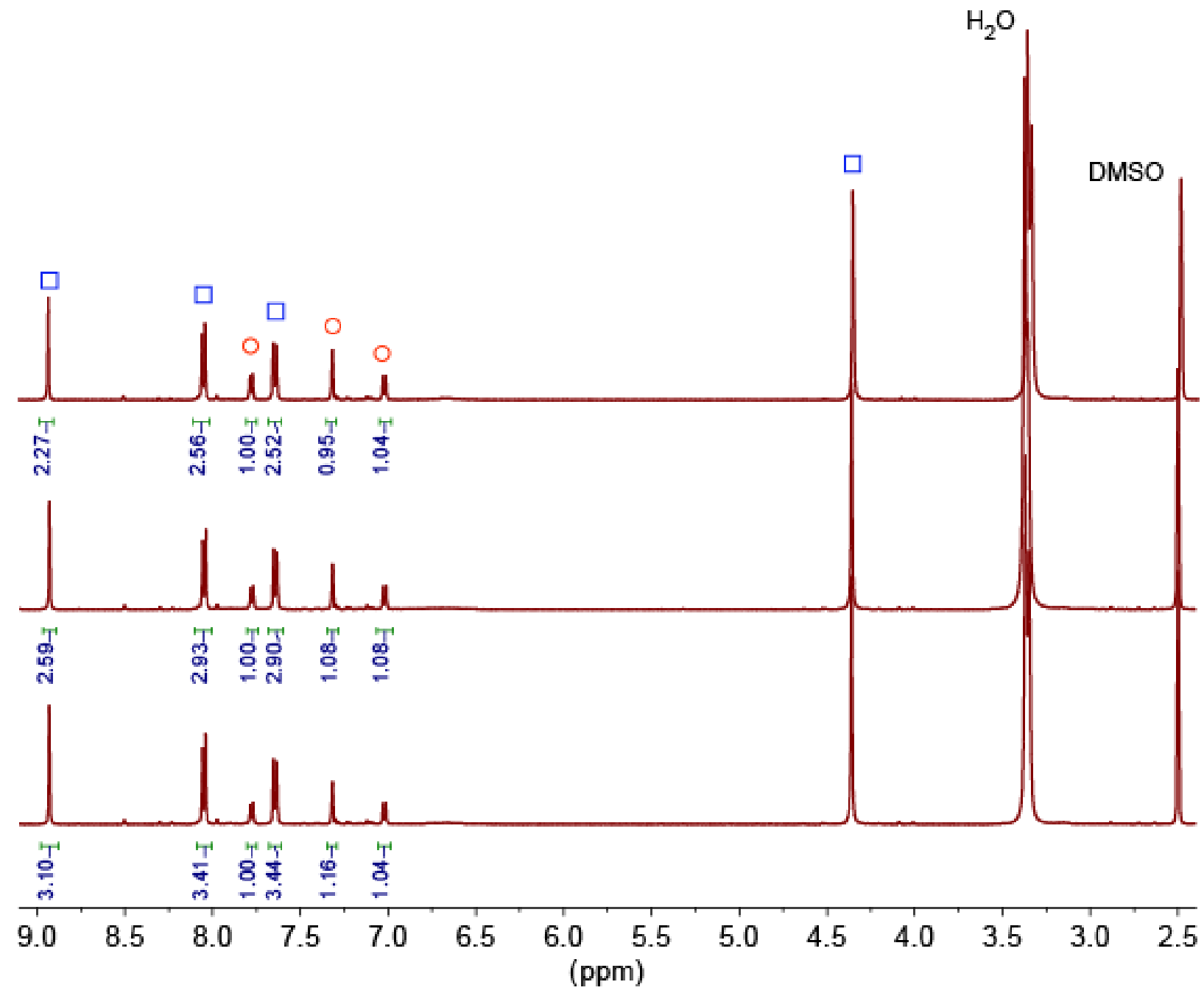

Figure S32. ${ }^{1} \mathrm{H}$ NMR spectra for three trials of digested IRMOF-3 modified with chloroacetyl isocyanate in chloroform for $6 \mathrm{~h}$. Red circles mark peaks related to unmodified IRMOF-3, and blue squares mark peaks related to IRMOF-3 after PSM with chloroacetyl isocyanate. Integration values for the determination of conversion percentage are taken from peaks at $7.78 \mathrm{ppm}$ for IRMOF-3 and $7.65 \mathrm{ppm}$ for IRMOF-3 after PSM. 


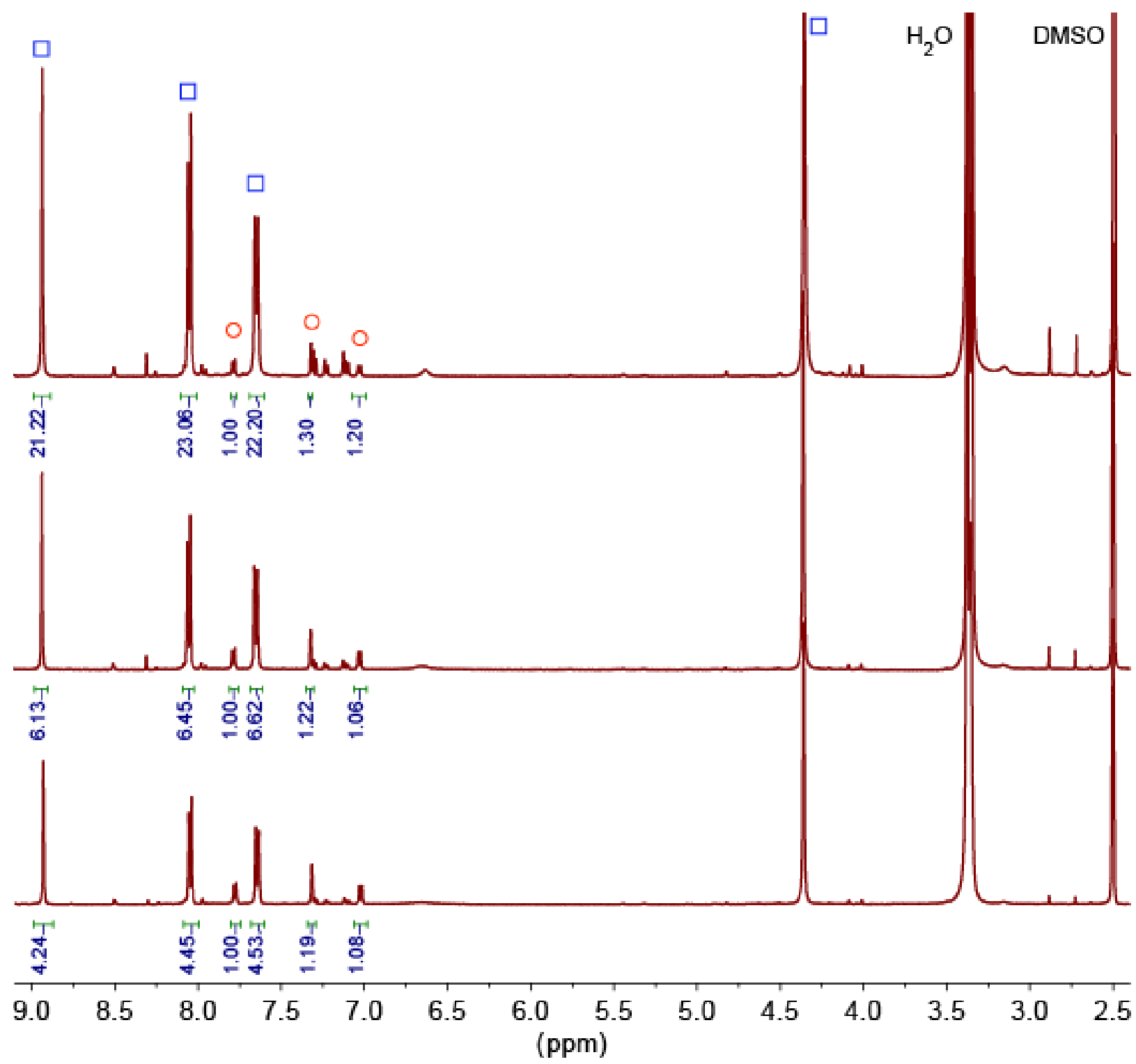

Figure S33. ${ }^{1} \mathrm{H}$ NMR spectra for three trials of digested IRMOF-3 modified with chloroacetyl isocyanate in chloroform for $9 \mathrm{~h}$. Red circles mark peaks related to unmodified IRMOF-3, and blue squares mark peaks related to IRMOF-3 after PSM with chloroacetyl isocyanate. Integration values for the determination of conversion percentage are taken from peaks at $7.78 \mathrm{ppm}$ for IRMOF-3 and $7.65 \mathrm{ppm}$ for IRMOF-3 after PSM. 


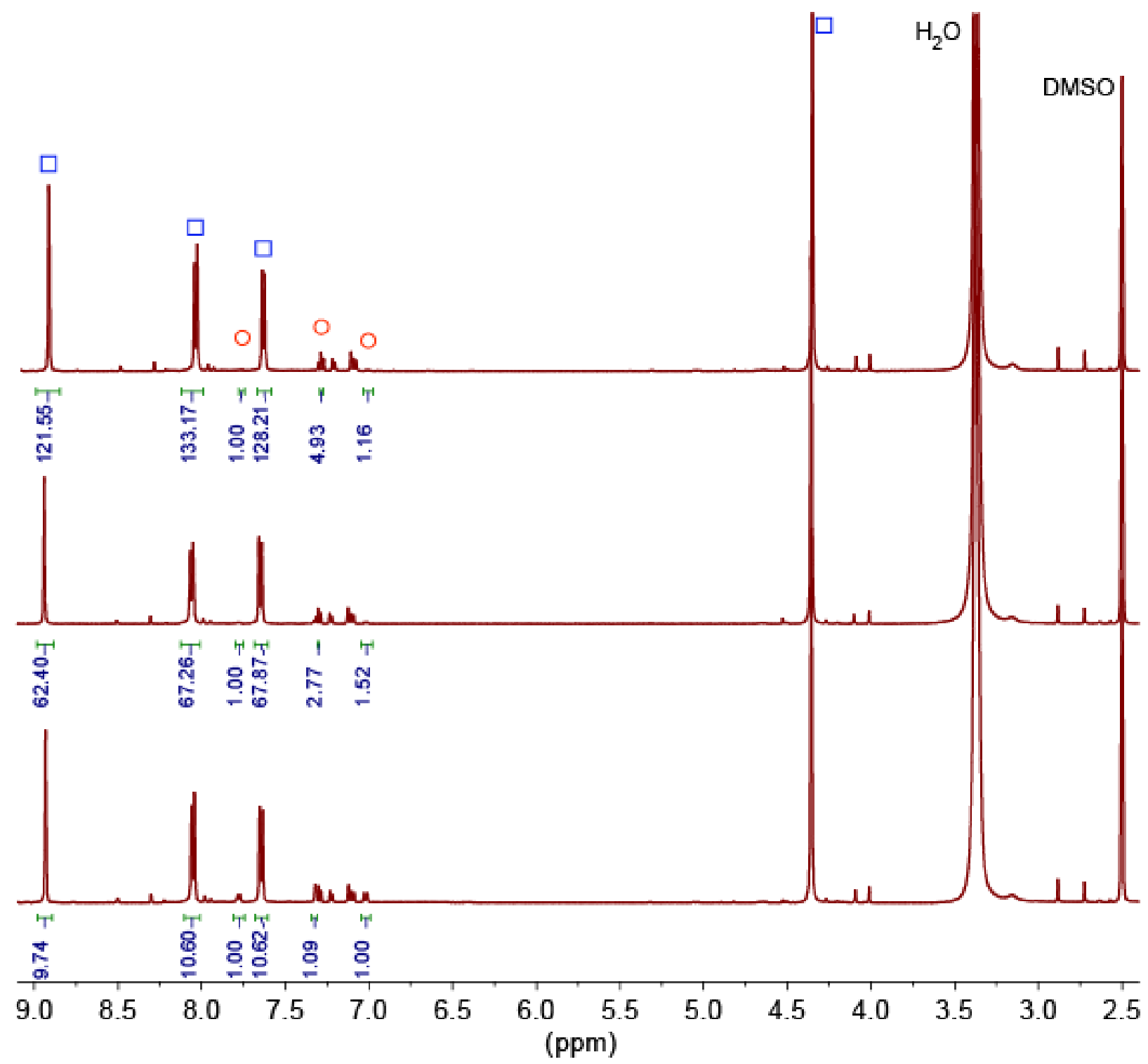

Figure S34. ${ }^{1} \mathrm{H}$ NMR spectra for three trials of digested IRMOF-3 modified with chloroacetyl isocyanate in chloroform for $24 \mathrm{~h}$. Red circles mark peaks related to unmodified IRMOF-3, and blue squares mark peaks related to IRMOF-3 after PSM with chloroacetyl isocyanate. Integration values for the determination of conversion percentage are taken from peaks at $7.78 \mathrm{ppm}$ for IRMOF-3 and $7.65 \mathrm{ppm}$ for IRMOF-3 after PSM. 


\section{Plots of PSM Conversion Calculated from ${ }^{1}$ H NMR Data Over Time}

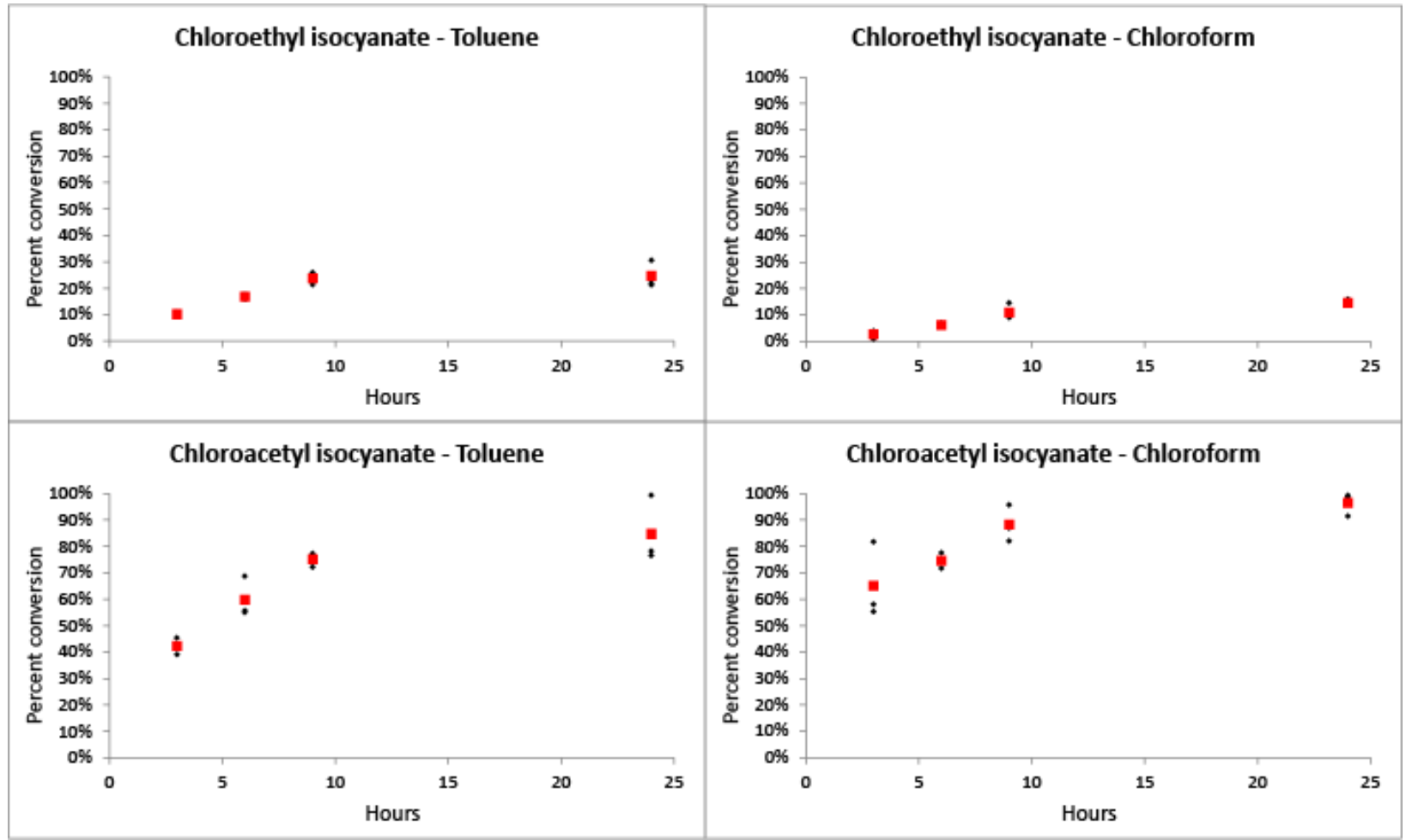

Figure S35. Plots derived from ${ }^{1} \mathrm{H}$ NMR data for the PSM of IRMOF-3 in various conditions where black dots indicate individual trial data and red squares indicate the average of the trial data. 

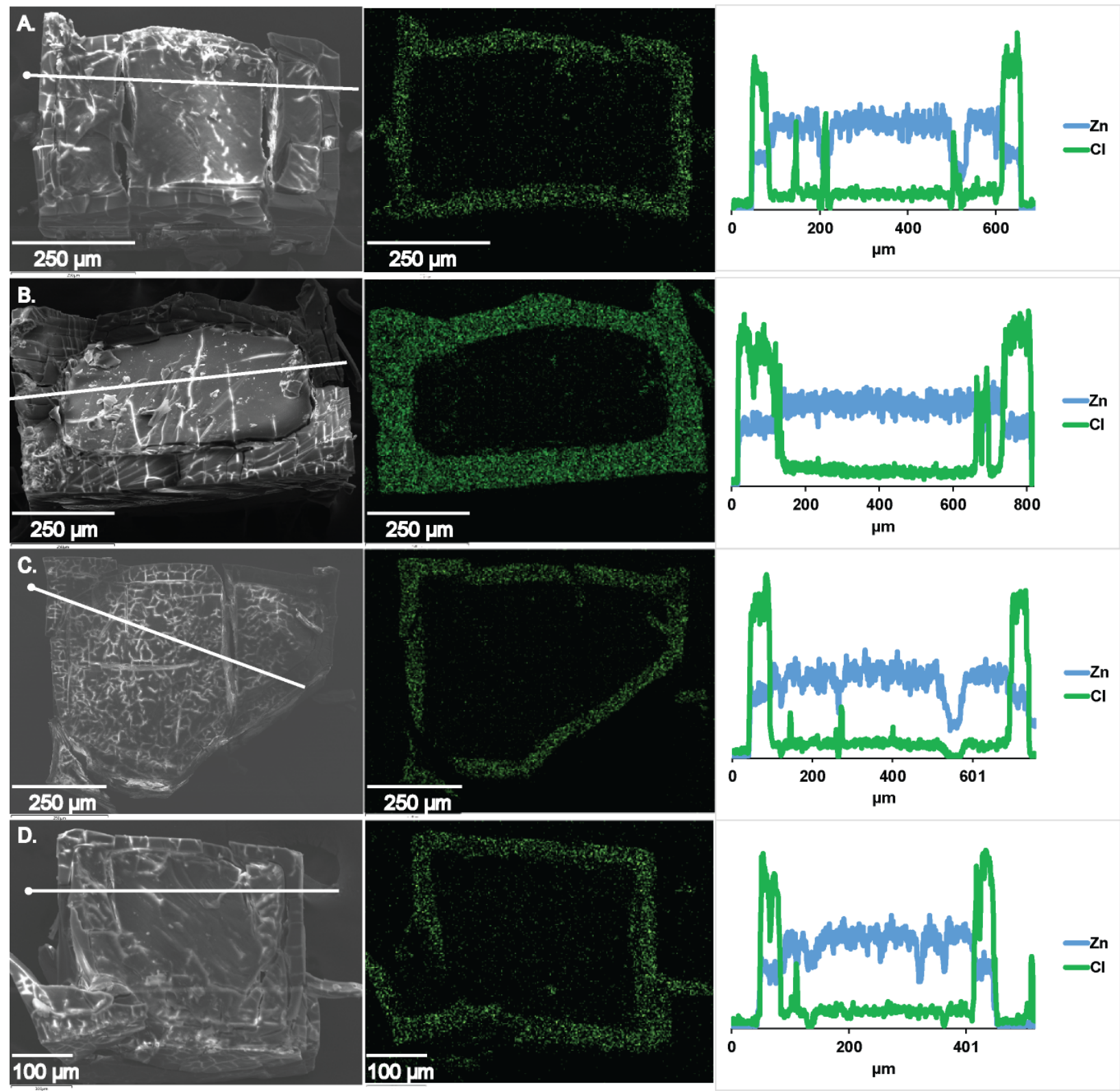

Figure S36. SEM images, EDS maps, and EDS linescans for PSM on IRMOF-3 with chloroacetyl isocyanate in A) carbon tetrachloride for 3 hours, B) trichloroethylene for 3 hours, C) 1,2-dichloroethane for 1 hour, and D) acetonitrile for 1 hour. 


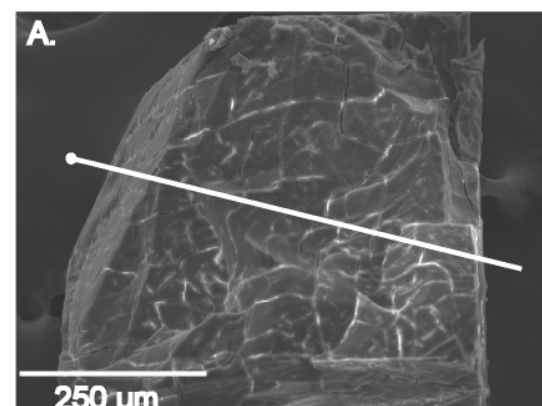

$250 \mathrm{~mm}$
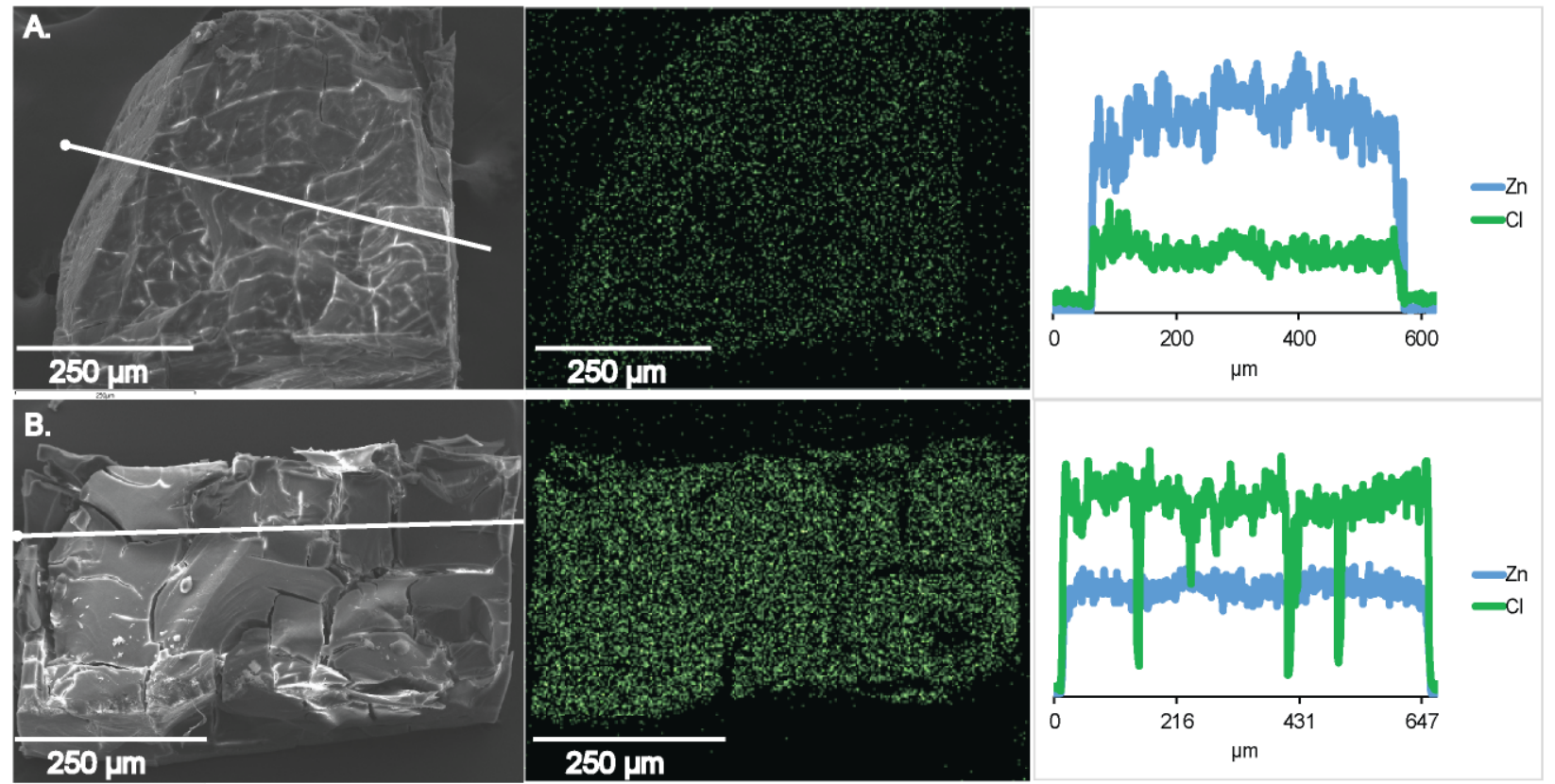

Figure S37. SEM images, EDS maps, and EDS linescans for PSM on IRMOF-3 with 2-chloroethyl isocyanate in carbon tetrachloride for A) 1 hour and B) 48 hours. 

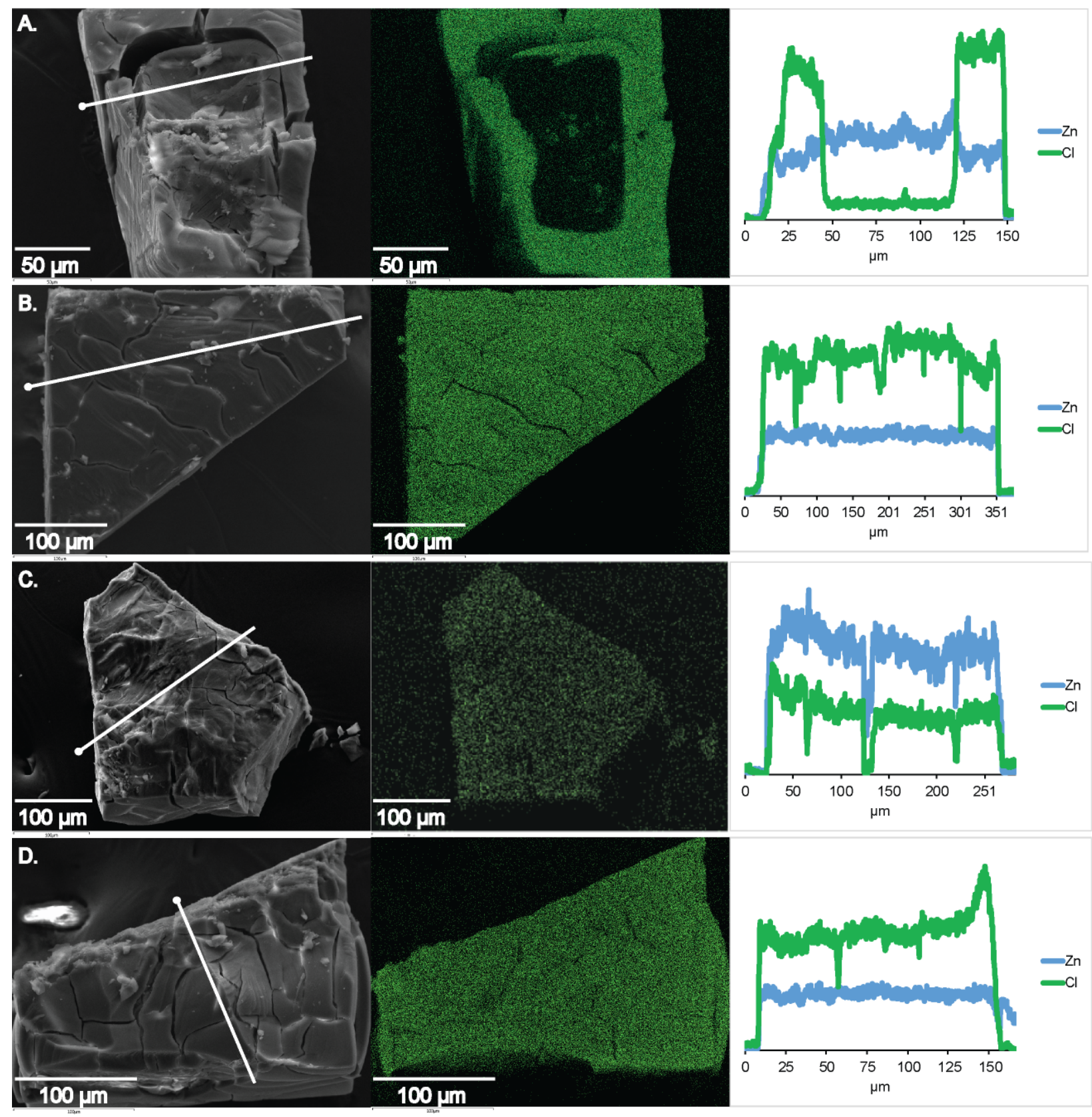

Figure S38. SEM images, EDS maps, and EDS linescans for PSM on IRMOF-3 in chloroform overnight with A) chloroacetyl isocyanate at $-18.8{ }^{\circ} \mathrm{C}$, B) chloroacetyl isocyanate at $50{ }^{\circ} \mathrm{C}$, showing a fully functionalized MOF C) 2-chloroethyl isocyanate at $-18.8^{\circ} \mathrm{C}$, and D) 2-chloroethyl isocyanate at $50{ }^{\circ} \mathrm{C}$. 

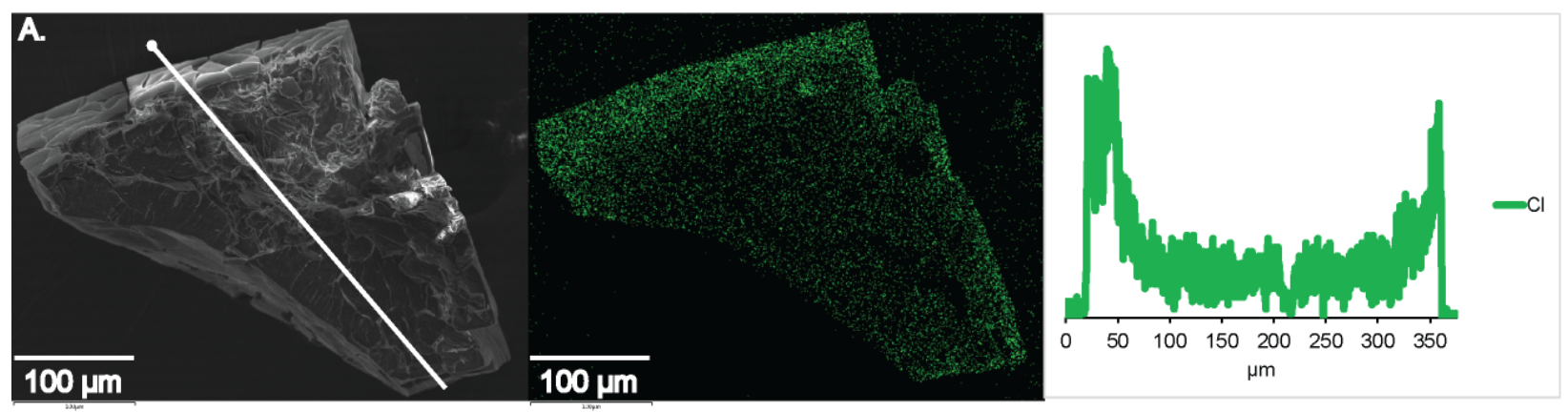

Figure S39. An SEM image, EDS map, and EDS linescan for PSM on IRMOF-3 with $500 \mathrm{mM} 2$ chloroethyl isocyanate in chloroform for 1 hour. The zinc signal is excluded for clarity. 


\section{References}

(1) Tanabe, K. K.; Wang, Z.; Cohen, S. M. Systematic Functionalization of a Metal-Organic Framework via a Postsynthetic Modification Approach. J. Am. Chem. Soc. 2008, 130, 8508-8517. 